\title{
Finite presentations for the mapping class group via the ordered complex of curves
}

\author{
Silvia Benvenuti \\ (Communicated by G. Gentili)
}

\begin{abstract}
We describe an algorithm to compute finite presentations for the mapping class group of a connected, compact, orientable surface, possibly with boundary and punctures. By an inductive process, such an algorithm, starting from a presentation well known for the mapping class group of the sphere and the torus with "few" boundary components and/or punctures, produces a presentation for the mapping class group of any other surface.
\end{abstract}

Key words. Mapping class group, complex of curves, inductive process.

2000 Mathematics Subject Classification. Primary 20F05; Secondary 20F36

\section{Introduction}

Let $F=F_{g, r}^{s}$ be a connected, compact, oriented surface of genus $g$ with $r$ boundary components and with a set $P=\left\{p_{1}, \ldots, p_{s}\right\}$ of $s$ distinguished points, called punctures $(g, r, s \geqslant 0)$. We denote by $\mathscr{H}(F, P)$ the group of orientation preserving homeomorphisms $h: F \rightarrow F$ which pointwise fix the boundary of $F$ and satisfy $h(P)=P$.

The mapping class group of $F$ is the group of the isotopy classes of elements of $\mathscr{H}(F, P)$ : we denote it by $\mathscr{M}(F)$, or by $\mathscr{M}_{g, r}^{s}$, as it clearly depends only (up to isomorphism) on the genus $g$, the number $r$ of boundary components and the number $s$ of punctures.

In this paper we will be concerned with the problem of finding finite presentations for $\mathscr{M}_{g, r}^{s}$.

By a result of Brown (see [4]), we can write down explicitly a finite presentation of a group $G$ acting on a simply connected simplicial complex $X$, provided we know:

- the structure of the 2 -skeleton of the quotient $X / G$, which must have a finite number of 2-cells;

- a finite presentation for the isotropy subgroup of a representative of every vertex in $X / G$;

- a finite set of generators for the isotropy subgroup of a representative of every edge in $X / G$.

Hence, the problem of finding a finite presentation for the mapping class group of 
a surface $F_{g, r}^{s}$ reduces to that of finding a suitable simply connected complex admitting an action of $\mathscr{M}_{g, r}^{s}$.

This line was implicitly followed by Hatcher and Thurston in [14]: in this paper the authors use a simplicial complex, the cut system complex, whose vertices are certain sets of $g$ circles on a closed, non-punctured surface $F$, to get an algorithm giving an explicit finite presentation for $\mathscr{M}_{g, 1}^{0}$, which unfortunately is rather complicated. Later, Harer [11] found a smaller complex with the same properties as the Hatcher-Thurston complex, and obtained a finite (but very unwieldy) explicit presentation for $\mathscr{M}_{g, 1}^{0}$; this presentation was then simplified by Wajnryb [31] in 1983. In 1998, starting from Wajnryb's result, Gervais [7] and Matsumoto [25] derived independently two simple presentations of $\mathscr{M}_{g .1}^{0}$ (it must be noticed that Gervais' result concerns more generally any $\mathscr{M}_{g, r}^{0}$ ). We recall also a recent paper by Wajnryb [32], where the author gives a completely self-contained proof of another simple presentation for the groups $\mathscr{M}_{g, 1}^{0}$ and $\mathscr{M}_{g, 0}^{0}$, still using the cut system complex; an interesting point of this paper is that the simple connectivity of the complex is shown by elementary methods.

Here, we apply Brown's method to a different complex, the ordered complex of curves, a suitable modification of the complex introduced by Harvey in [13], and deeply studied by several authors (see for example [12, 18, 23, 24, 27, 28]). We get this way, as a byproduct, a simpler and more direct proof of the presentations given in $[14,31,7]$. Moreover, our method works for the general case of $\mathscr{M}_{g, r}^{s}$.

During the preparation of this paper, we were informed of a paper by Hirose [15], where the author recovers Gervais' presentation exploiting the action of the mapping class group on a different "complex of curves", involving only non-separating curves and simplices. We remark here that the main advantage in using our complex of curves, instead of the Hatcher-Thurston's one or the one used by Hirose, is the fact that our complex, though having several $\mathscr{M}_{g, r}^{s}$-equivalence classes of vertices (while the others have a single such class), has only triangular 2-cells, which makes it particularly powerful in reducing the presentation coming from Brown's method to a very simple and meaningful one.

The paper is organized as follows: in Section 2 we state a special version of Brown's result which applies to our case. In Section 3 we define the ordered complex of curves $X_{g, r, s}^{\text {ord }}$, and show that, with the exception of a finite number of cases, called sporadic cases, such a complex is simply connected and admits a natural action of $\mathscr{M}_{g, r}^{s}$ satisfying all the hypotheses needed to apply Brown's Theorem. To be precise, the sporadic cases are the surfaces $F_{g, r}^{s}$ with $g=0, r+s=0,1,2,3,4,5$ and $g=1$, $r+s=0,1,2$, and their presentations are well known (see Section 5). In Section 4 we analyze the 2-skeleton of $X_{g, r, s}^{\text {ord }}$ and explain how to produce a finite presentation for the isotropy subgroups of its vertices and edges, provided one knows a presentation for each $\mathscr{M}_{g^{\prime}, r^{\prime}}^{s^{\prime}}$ such that $\left(g^{\prime}, r^{\prime}+s^{\prime}\right)<(g, r+s)$ (with the lexicographic order). Hence, the method of Section 2 recursively produces a presentation of any nonsporadic $\mathscr{M}_{g, r}^{s}$, provided we start with a presentation for each sporadic case. In Section 5 we explain such an inductive process and, for the sake of completeness, we recall a presentation for the non-punctured sporadic cases, that is the basis of the induction in the situations treated in the last two sections. Section 6 is devoted to the detailed 
analysis of the non-punctured case. Let $V$ be the set of $\mathscr{M}_{g, r}^{s}$-equivalence classes of simple generic closed curves on $F_{g, r}^{s}$, not isotopic to boundary components. Moreover, let $E$ be the set of $\mathscr{M}_{g, r}^{s}$-equivalence classes of pairs of disjoint, not isotopic, simple generic closed curves, not isotopic to boundary components. We prove the following result:

Theorem 1. If $F_{g, r}^{0}$ is not sporadic, there exists a choice of a representative $s(v)$ for every class $v \in V$ and of a representative $s(e)$ for every class $e \in E$, such that $\mathscr{M}\left(F_{g, r}^{0}\right)$ is the free product of the isotropy subgroups of all the $s(v)$ 's, amalgamated along the isotropy subgroups of the $s(e)$ 's.

Since it is possible to find a presentation for the isotropy subgroup of a curve $s(v)$ starting from the knowledge of a presentation for the mapping class group of the surface obtained from $F_{q, r}^{0}$ cutting it open along $s(v)$, if we apply recursively this theorem to all the non-sporadic subsurfaces $F_{g^{\prime}, r^{\prime}}^{0}$ obtained from $F_{g, r}^{0}$ cutting them open along generic simple closed curves, we may conclude that all the relations needed to present the mapping class group are supported in subsurfaces homeomorphic to sporadic surfaces.

Finally, in Section 7 we show that we recover Gervais' presentation [7] for the mapping class group of any non-sporadic surface, provided we start the inductive process with the Gervais presentation for the sporadic subsurfaces. Analogously, once we have the presentations for the sporadic surfaces according to some "style" (e.g. in terms of Dehn twists [7], or as quotients of Artin groups [25, 20]), our method produces a presentation of the same "style" for every $F_{g, r}^{s}$. We may then say that the complex of curves allows to recover by a unique algorithm at least all the simpler known presentations. Actually, our analysis also suggests the existence of other simple presentations, where the generators are, besides a number of Dehn twists, a family of elements having an intrinsic geometric meaning. We will describe these aspects in a forthcoming paper.

For the reader's convenience, we recall in the Appendix the definition of Dehn twist, braid twist and semitwist.

\section{Description of the general method}

In [4] Brown describes a general method to get a presentation for a group $G$ looking at its action on a simply connected CW-complex $X$ (see also [10] and [3], where similar results are discussed in the setting of small categories without loops and complexes of groups). We describe in this section a particular case of Brown's theorem, under some additional hypotheses both on the complex and on the action. We refer the reader to [4], [10], or [3] for the details.

Let $X$ be a CW-complex with oriented edges. We denote by $E(X)$ the set of edges of $X$, and by $V(X)$ the set of vertices. We define two maps

$$
i, t: E(X) \rightarrow V(X)
$$


giving respectively the initial and terminal vertex $i(e)$ and $t(e)$ of the edge $e$, according to the given orientation. We suppose each oriented edge is determined by its ends, and we denote by $\left\langle v_{1}, v_{2}\right\rangle$ the edge joining $v_{1}$ and $v_{2}$, with $i\left(\left\langle v_{1}, v_{2}\right\rangle\right)=v_{1}$ and $t\left(\left\langle v_{1}, v_{2}\right\rangle\right)=v_{2}$; sometimes, in this situation, we will write also $v_{1}<v_{2}$.

Let $G$ be a group acting by homeomorphisms on $X$.

Definition 2. The action of $G$ on $X$ is called good and orientation compatible (or shortly $X$ is a good $G$-CW-complex) if

(i) the action of $G$ permutes cells of the same dimension;

(ii) if $g \in G$ leaves a cell invariant, then its restriction to that cell is the identity;

(iii) $g(i(e))=i(g(e))$ and $g(t(e))=t(g(e))$ for each $e \in E(X)$ and for each $g \in G$.

Given a good $G$-CW-complex $X$, we denote by $\bar{X}$ the quotient space $X / G$, and by $\pi: X \rightarrow \bar{X}$ the projection.

We suppose that the closure of each 2-cell of $X$ is an embedded triangle, that is a subcomplex with the induced canonical cell decomposition. Moreover, we suppose that each triangle is determined by its oriented edges, and that the orientation of the edges is such that no triangle has an oriented loop as a boundary; in these hypotheses, we label by $f, m, p$ the vertices of a 2-cell $T$, where $f<m<p$, and we label by $a, b, c$ the edges of $T$, where $a=\langle f, m\rangle, b=\langle m, p\rangle, c=\langle f, p\rangle$.

We first consider the following choices:

(1) for every $v \in V(\bar{X})$, we choose a representative $s(v) \in V(X)$ (that is $\pi(s(v))=v$ ), and we denote by $G_{v}$ the isotropy subgroup of $s(v)$,

$$
G_{v}=\operatorname{Stab}(s(v))
$$

(2) for every $e \in E(\bar{X})$, we choose a representative $s(e) \in E(X)$ (that is $\pi(s(e))=e$ ), we denote by $G_{e}$ the isotropy subgroup of $s(e)$, and we choose two elements $\gamma_{e, i}, \gamma_{e, t} \in G$ such that

$$
\gamma_{e, i}(i(s(e)))=s(i(e)), \quad \gamma_{e, t}(t(s(e)))=s(t(e))
$$

(see Figure 2.1);

(3) for every $T \in \bar{X}_{2}$ (the 2-skeleton of $\bar{X}$ ), we choose a representative $s(T) \in X_{2}$ (that is $\pi(s(T))=T)$. Moreover, we choose, for every $T \in \bar{X}_{2}$, the elements $\gamma_{T, a}, \gamma_{T, b}$, $\gamma_{T, c}$ of $G$ such that

$$
\gamma_{T, e}(\tilde{e})=s(e), \quad e \in\{a, b, c\}
$$

where the tilde denotes the lifting in $s(T)$ of a vertex (or edge) in $T$ (see Figure $2.1)$;

(4) we choose a maximal tree $\mathscr{T}$ in the 1 -skeleton $\bar{X}_{1}$.

Then, we have the following result. 

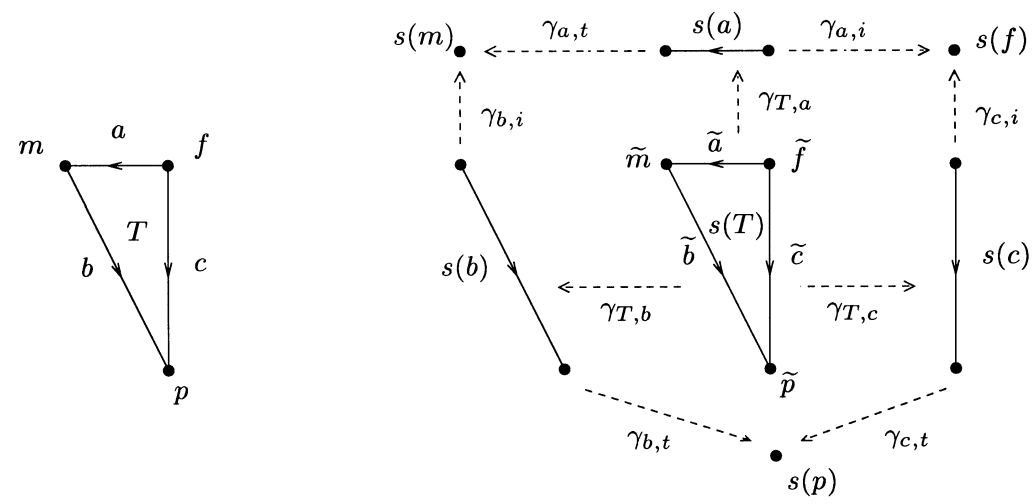

Figure 2.1. A 2-cell of $\bar{X}$ (left-hand picture), its representative in $X$ and the meaning of the elements $\gamma_{e, i}, \gamma_{e, t}$ and $\gamma_{T, e}$ (right-hand picture).

Theorem 3. Let us suppose that:

(i) $X$ is simply connected;

(ii) the isotropy subgroup of each vertex $v$ is finitely presented, $G_{v}=\left\langle S_{v} \mid R_{v}\right\rangle$;

(iii) the isotropy subgroup of each edge e is finitely generated, Gen $G_{e}=S_{e}$;

(iv) the quotient $\bar{X}=X / G$ has a finite 2-skeleton.

Then $G$ is finitely presented, and a presentation of $G$ is given by

$$
G=\left\langle\bigcup_{v \in V(\bar{X})} S_{v} \cup \bigcup_{e \in E(\bar{X})} E \mid \bigcup_{v \in V(\bar{X})} R_{v} \cup R^{(2)} \cup R^{(3)} \cup R^{(4)}\right\rangle,
$$

where $E$ is a symbol, associated to the edge e, for each $e \in E(\bar{X})$, and

$$
\begin{aligned}
& R^{(2)}=\{E=1 \mid e \in \mathscr{T}\} ; \\
& R^{(3)}=\left\{E \gamma_{e, t} g \gamma_{e, t}^{-1} E^{-1}=\gamma_{e, i} g \gamma_{e, i}^{-1} \mid g \in S_{e}, e \in E(\bar{X})\right\} ; \\
& R^{(4)}=\left\{\gamma_{c, i} \gamma_{T, c} \gamma_{T, a}^{-1} \gamma_{a, i}^{-1} A \gamma_{a, t} \gamma_{T, a} \gamma_{T, b}^{-1} \gamma_{b, i}^{-1} B \gamma_{b, t} \gamma_{T, b} \gamma_{T, c}^{-1} \gamma_{c, t}^{-1}=C \mid T \in \bar{X}_{2}\right\} .
\end{aligned}
$$

Here and in the following we use the capital letter to indicate the generator associated to the edge denoted with the corresponding lowercase letter (for instance, $A$ is the symbol corresponding to the edge a).

Remark 4. As we said at the beginning of the section, this is a particular case of a general result by Brown, which holds without the assumptions (ii) and (iii) on the action of $G$, and without restrictive hypotheses on the shape and the boundary 
orientation of the 2-cells of $X$. We stated the result in this weaker form because these hypotheses simplify the statements, and are satisfied in our situation. In particular, if we do not make the hypothesis on the shape of the 2-cells, we still get a presentation of the kind described above, but the relations $R^{(4)}$ are in general much more complicated. We will see in Section 5 that the hypothesis on the 2-cells is central in further simplifying this presentation.

\section{The ordered complex of curves}

Let $F=F_{g, r}^{s}$ be a connected, compact, oriented surface of genus $g$, with $r$ boundary components and $s$ punctures, $r, s \geqslant 0$; we denote by $P$ the set of punctures of $F$. A simple closed curve in $F \backslash P$ is an embedding $\gamma: \mathbb{S}^{1} \rightarrow F \backslash P$ which does not intersect the boundary of $F$, and two simple closed curves $\alpha, \beta$ are said to be isotopic $(\alpha \simeq \beta)$ if there exists a continuous family $h_{t} \in \mathscr{H}(F, P), t \in[0,1]$ such that $h_{0}$ is the identity and $h_{1} \circ \alpha=\beta$. A curve is called generic if its image does not bound a disk or a disk with one puncture.

Definition 5. The complex of curves on $F$ is the simplicial complex $X=X_{g, r}^{s}$ of dimension $3 g-4+r+s$ whose $k$-simplices are the isotopy classes of families $\alpha=$ $\left\{\alpha_{0}, \ldots, \alpha_{k}\right\}$ of $k+1$ generic simple closed curves in $F \backslash P$ satisfying the following conditions:

(i) $\alpha_{i} \cap \alpha_{j}=\varnothing$

if $i \neq j$;

(disjoint)

(ii) $\alpha_{i} \not \alpha_{j}$ and $\alpha_{i} \not \alpha_{j}^{-1}$

if $i \neq j$;

(pairwise not isotopic)

(iii) $\alpha_{i} \not \longleftarrow$ any boundary component of $F$

for all $i$. (not isotopic to boundary components)

We call such a family a generic $(k+1)$-family of closed curves.

Notice that the curves are not oriented, and that the families we consider are not ordered, i.e. the two families $\alpha=\left\{\alpha_{0}, \ldots, \alpha_{k}\right\}$ and $\beta=\left\{\beta_{0}, \ldots, \beta_{k}\right\}$ are equivalent (i.e. represent the same $k$-simplex in $X$ ) if there exists a permutation $\sigma \in \Sigma_{k+1}$ such that $\alpha_{i} \simeq \beta_{\sigma(i)}^{ \pm 1}$ for every $i \in\{0, \ldots, k\}$. We denote by $X_{k}$ the $k$-skeleton of $X$ and by $[\alpha]=\left[\alpha_{0}, \ldots, \alpha_{k}\right]$ the simplex represented by the family $\alpha=\left\{\alpha_{0}, \ldots, \alpha_{k}\right\}$.

Theorem 6. If $g \geqslant 1, r+s \geqslant 1$, then $X_{g, r}^{s}$ is $(2 g+r+s-4)$-connected. Moreover, $X_{g, 0}^{0}$ is $(2 g-3)$-connected and $X_{0, r}^{s}$ is $(r+s-5)$-connected.

For the proof of this theorem we refer the reader to Harer [12], where the result is proven in the setting of Thurston train tracks theory, or to Ivanov [18], where the same result is proven using Cerf theory. We recall that the 1 -connectedness of $X$ for $g \geqslant 2$ was first proved by Ivanov in [17], where it is derived from the 1-connectedness of the complex of Hatcher-Thurston; instead, the proofs in [18] and [21] are independent from that. 


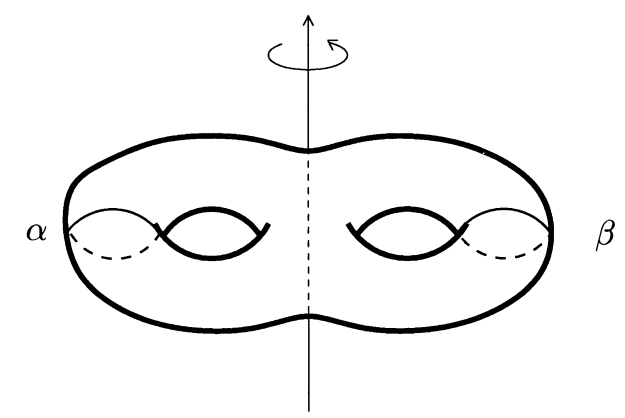

Figure 3.1. An element of $\mathscr{M}_{2,0}^{0}$ that leaves a 1-cell invariant but interchanges its endpoints.

In particular, except for the cases $g=0, r+s=0,1,2,3,4,5$ and $g=1, r+s=$ $0,1,2$ that we call sporadic, the complex of curves $X_{g, r}^{s}$ is simply connected (see also the sketch of a simple proof in [19]).

In order to have an orientation for the edges of $X$, we fix a total ordering for its vertices and we orient each edge accordingly. When we consider oriented edges, or more generally oriented $k$-simplices, we use the notation $\left\langle\alpha_{0}, \ldots, \alpha_{k}\right\rangle$ with the meaning $\alpha_{0}<\alpha_{1}<\cdots<\alpha_{k}$.

If $\alpha \simeq \beta$ and $h_{1}, h_{2} \in \mathscr{H}(F, P)$ are isotopic, then clearly $h_{1}(\alpha) \simeq h_{2}(\beta)$; therefore the mapping class group $\mathscr{M}(F)$ acts on the set of isotopy classes of simple closed curves, i.e. on $X_{0}$, and this action naturally extends to the $k$-skeleton of $X$. Unfortunately this action is neither good nor orientation compatible, as one can see immediately from the following example: we consider $F_{2,0}^{0}$, with $\alpha$ and $\beta$ as in Figure 3.1. The rotation of 180 degrees around the $z$-axis globally fixes the 1 -simplex $[\alpha, \beta]$, but interchanges $[\alpha]$ and $[\beta]$, and therefore its restriction to $[\alpha, \beta]$ is not the identity.

To overcome this problem, we consider another complex, the ordered complex of curves.

Definition 7. The ordered complex of curves on $F$ is the simplicial complex, that we denote by $X^{\text {ord }}$, whose $k$-simplices are the isotopy classes of ordered families of $k+1$ generic simple closed curves satisfying the conditions (i), (ii), (iii) of Definition 5.

The complex $X$ with its orientation can be clearly seen as a subcomplex of $X^{\text {ord }}$ (see Figure 3.2). The action of $\mathscr{M}(F, P)$ on $X$ extends to a good and orientation compatible action on $X^{\text {ord }}$ : namely, if $[\alpha, \beta]$ is a non-oriented edge of $X$ whose image under $g \in \mathscr{M}(F, P)$ is $[g(\alpha), g(\beta)]$, we set

$$
g(\langle\alpha, \beta\rangle)=\langle g(\alpha), g(\beta)\rangle
$$

This can be done since both $\langle g(\alpha), g(\beta)\rangle$ and $\langle g(\beta), g(\alpha)\rangle$ belong to $X^{\text {ord }}$. Hence, we have a good $G$-simplicial complex $X^{\text {ord }}$ (from now on $G$ will denote the mapping class group, unless otherwise stated), and it remains to show that it is simply connected. 
$X$

$\stackrel{1-1}{\longleftrightarrow}$
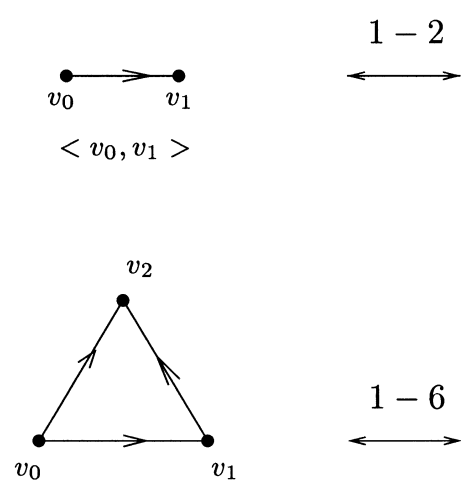

$<v_{0}, v_{1}, v_{2}>$

$1-6$

$$
X^{\text {ord }}
$$

$v_{0}$
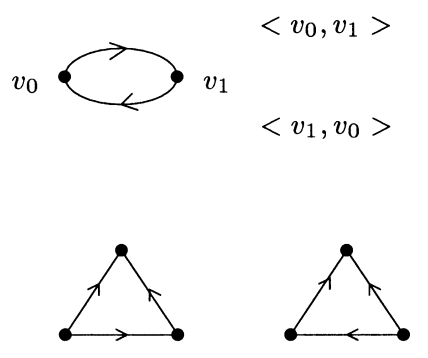

$<v_{1}, v_{0}, v_{2}>$
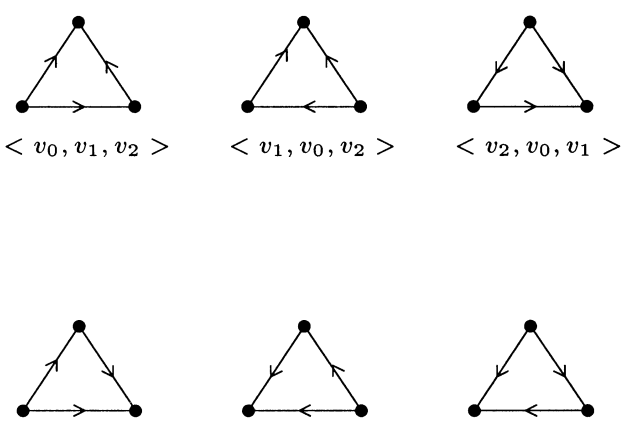

$<v_{0}, v_{1}, v_{2}>$

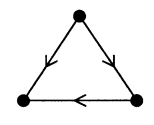

$<v_{2}, v_{1}, v_{0}>$

Figure 3.2. The ordered complex of curves.

Proposition 8. If $X$ is simply connected, then $X^{\text {ord }}$ is also simply connected.

Proof. It is sufficient to show that all the loops of type

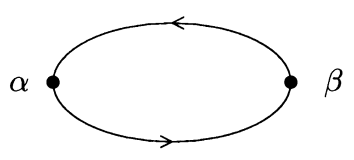

in $X_{1}^{\text {ord }}$ are contractible in $X^{\text {ord }}$ if this is true, each time that a loop in $X_{1}^{\text {ord }}$ contains an edge of $X_{1}^{\text {ord }} \backslash X_{1}$, we can substitute it with the corresponding edge of $X_{1}$, thus we are done by the simple connectivity of $X$. Supposing $\alpha<\beta$ in the chosen ordering of the vertices of $X$ and supposing there is a $\gamma \in X_{0}$ such that $\alpha, \beta$ and $\gamma$ are the vertices of a triangle in $X_{2}$, the situation is one of the three described in Figure 3.3. In any case, in $X_{2}^{\text {ord }}$ we have two triangles that allow us to contract the loop $\alpha \beta \alpha$ to a point (such triangles are respectively $\langle\gamma, \alpha, \beta\rangle$ and $\langle\gamma, \beta, \alpha\rangle,\langle\alpha, \beta, \gamma\rangle$ and $\langle\beta, \alpha, \gamma\rangle,\langle\gamma, \alpha, \beta\rangle$ and $\langle\gamma, \beta, \alpha\rangle$ ). Finally, we conclude noticing that in the non-sporadic cases such a $\gamma$ 


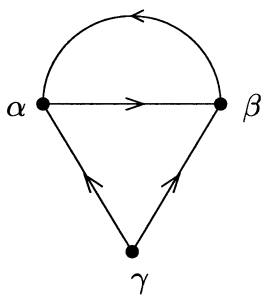

if $\gamma<\alpha<\beta$

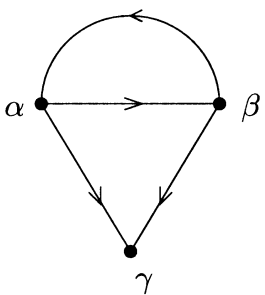

if $\alpha<\beta<\gamma$

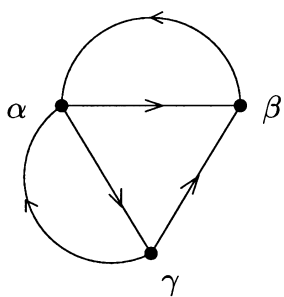

if $\alpha<\gamma<\beta$

Figure 3.3. Contracting a loop $\alpha \beta \alpha$.

always exists, because given two simple closed generic curves, disjoint and not isotopic, it is always possible to complete them to a pants decomposition (see Subsection 4.1), hence obtaining a third simple closed generic curve which is disjoint and not isotopic to the other two.

\section{The action of $\mathscr{M}_{g, r}^{s}$ on $X^{\text {ord }}$}

In the previous section we showed that in the non-sporadic cases the ordered complex of curves $X^{\text {ord }}=X_{g, r, s}^{\text {ord }}$ of a surface $F=F_{g, r}^{s}$ satisfies the hypotheses of Theorem 3; now we give the main tools to algorithmically carry on the method. More precisely we describe

(i) how to construct $\bar{X}_{2}^{\text {ord }}$, the 2-skeleton of the quotient $X^{\text {ord }} / \mathscr{M}_{g, r}^{s}$;

(ii) how to find a finite presentation for the isotropy subgroup of a vertex $v \in V\left(X^{\text {ord }}\right)$ and how to find a finite set of generators for the isotropy subgroup of an edge $e \in E\left(X^{\text {ord }}\right)$.

4.1 The structure of $\bar{X}_{2}^{\text {ord }}$. Let us come back to the non-ordered complex of curves $X$ : we want to find a method to determine whether two classes $\alpha, \beta \in X$ are in the same $\mathscr{M}_{g, r}^{s}$-orbit, and to find a representative for each $\mathscr{M}_{g, r}^{s}$-orbit. Let $\alpha=\left\{\alpha_{1}, \ldots, \alpha_{k}\right\}$ be a generic $k$-family of closed curves on a punctured surface $F=F_{g, r}^{s}$. We denote by $F_{\alpha}$ the natural compactification of $F \backslash\left(\bigcup_{i=1}^{k} \alpha_{i}\right)$, and by $\rho_{\alpha}: F_{\alpha} \rightarrow F$ the continuous map induced by the inclusion of $F \backslash\left(\bigcup_{i=1}^{k} \alpha_{i}\right)$ in $F$. Let $N$ be a connected component of $F_{\alpha}$, and $\gamma: \mathbb{S}^{1} \rightarrow \partial N$ a boundary curve of $N$. We say that $\gamma$ is an exterior boundary curve of $N$ if $\rho_{\alpha} \circ \gamma$ is a boundary component of $F$. For each curve $\alpha_{i}: \mathbb{S}^{1} \rightarrow F$ in the family $\alpha$ there are two distinct boundary curves $\gamma, \gamma^{\prime}: \mathbb{S}^{1} \rightarrow \partial F_{\alpha}$ such that $\rho_{\alpha} \circ \gamma=\rho_{\alpha} \circ \gamma^{\prime}=\alpha_{i}$, and two situations are possible: either $\gamma$ and $\gamma^{\prime}$ are boundary curves of the same connected component $N$ of $F_{\alpha}$ (in that case we say that $\alpha_{i}$ is a non-separating limit curve of $N$ ), or $\gamma$ is a boundary component of $N$ and $\gamma^{\prime}$ is a boundary component of a different connected component $N^{\prime}$ (in that case we say that $\alpha_{i}$ is a separating limit curve of $N$ and $N^{\prime}$ ) (see Figure 4.1).

We are now able to state the following proposition, whose proof is trivial: 


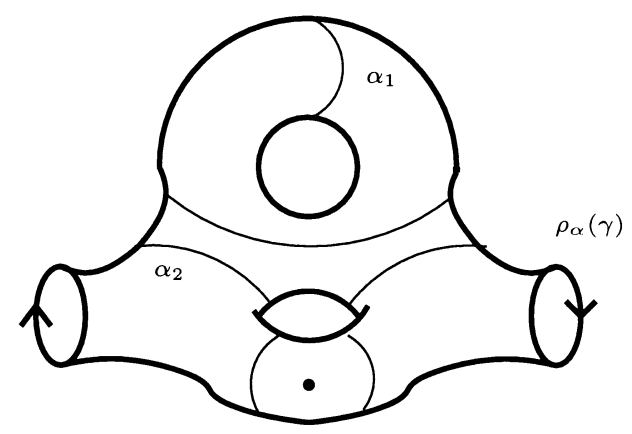

Figure 4.1. Exterior boundary curves $(\gamma)$, separating limit curves $\left(\alpha_{2}\right)$ and non-separating limit curves $\left(\alpha_{1}\right)$.

Proposition 9. If $[\alpha]=\left[\alpha_{1}, \ldots, \alpha_{k}\right]$ and $[\beta]=\left[\beta_{1}, \ldots, \beta_{h}\right]$ are two simplices of $X$, then $[\alpha]$ is $\mathscr{M}_{g, r}^{s}$-equivalent to $[\beta]$ if and only if

(1) $k=h$;

(2) there exists a one to one correspondence between the components of $F_{\alpha}$ and those of $F_{\beta}$;

(3) there exists a permutation $\sigma \in \Sigma_{k}$ such that, for every pair $\left(N, N^{\prime}\right)$ where $N$ is any component of $F_{\alpha}$ and $N^{\prime}$ the corresponding component of $F_{\beta}$, we have:

- $g(N)=g\left(N^{\prime}\right), s(N)=s\left(N^{\prime}\right), r(N)=r\left(N^{\prime}\right)$, where we denote by $g(N)$ the genus, by $s(N)$ the number of punctures and by $r(N)$ the number of boundary components of $N$;

- if $\gamma$ is an exterior boundary curve of $N$ there exist an exterior boundary curve $\gamma^{\prime}$ of $N^{\prime}$ such that $\rho_{\alpha} \circ \gamma=\rho_{\beta} \circ \gamma^{\prime}$;

- if $\alpha_{i}$ is a separating limit curve of $N$, then $\beta_{\sigma(i)}$ is a separating limit curve of $N^{\prime}$;

- if $\alpha_{i}$ is a non-separating limit curve of $N$, then $\beta_{\sigma(i)}$ is a non-separating limit curve of $N^{\prime}$.

Now, in order to find a family of representatives for $\mathscr{M}_{g, r}^{s}$-orbits in $X$, we introduce the notion of pants decomposition.

A pair of pants of type $I$ is a (surface homeomorphic to a) disk with 2 punctures (i.e. $F_{0,1}^{2}$ ), a pair of pants of type $I I$ is an annulus with one puncture (i.e. $F_{0,2}^{1}$ ), and a pair of pants of type III is a sphere with 3 holes (i.e. $F_{0,3}^{0}$ ).

We say that the family $\alpha$ determines a pants decomposition of $F$ if each component $N$ of $F_{\alpha}$, with set of punctures $N \cap \rho_{\alpha}^{-1}$ \{punctures of $F$, is a pair of pants (see Figure 4.3). Then, it is easy to check that $F_{g, r}^{s}$ admits a pants decomposition, provided that

$$
(g, r, s) \notin\{(0,0,0),(0,0,1),(0,0,2),(0,0,3),(0,1,0),(0,1,1),(0,2,0),(1,0,0)\} .
$$

In particular every non-sporadic surface admits a pants decomposition. 


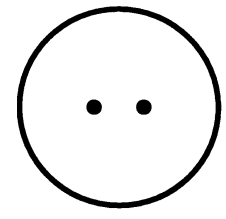

Type I

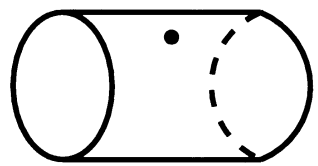

Type II

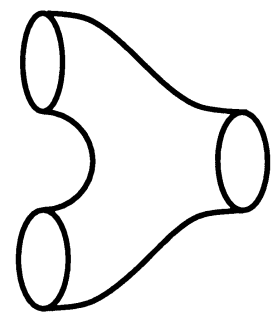

Type III

Figure 4.2. Pair of pants.

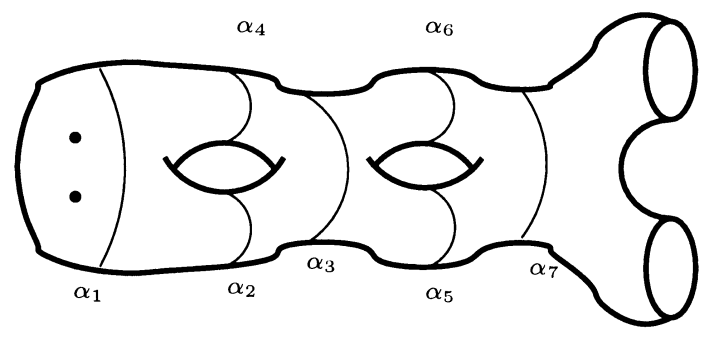

Figure 4.3. A pants decomposition.

A generic $k$-family determines a pants decomposition of $F$ if and only if $k=$ $3 g+r+s-3$, i.e. if and only if such a family represents a simplex of maximal dimension in the complex of curves; moreover, the number of pants in any decomposition is $2 g+r+s-2$.

Given a generic $k$-family $\alpha=\left\{\alpha_{1}, \ldots, \alpha_{k}\right\}$, it can be proven that we can always complete it to a pants decomposition of $F$, i.e. there exist generic closed curves $\left\{\alpha_{k+1}, \ldots, \alpha_{3 g+r+s-3}\right\}$ such that $\left\{\alpha_{1}, \ldots, \alpha_{3 g+r+s-3}\right\}$ determines a pants decomposition of $F$. Hence, to find the representatives of the $\mathscr{M}_{g, r}^{s}$-orbits of $X$ we need to look at the subfamilies of the pants decompositions, more precisely:

(1) we take the disjoint union $\tilde{F}$ of $n_{i}$ pants of type $i, n_{I}+n_{I I}+n_{I I I}=2 g+r+s-2$, $2 n_{I}+n_{I I}=s$, we choose $3 g+r+s-3$ boundary curves of $\tilde{F}$ and we glue the connected components of $\tilde{F}$ identifying the curves of each pair: the identification space we obtain is homeomorphic to $F$, the set of $3 g+r+s-3$ curves which are the projection of the chosen pairs is a pants decomposition of this space, and every $\mathscr{M}_{g, r}^{s}$-orbit of pants decompositions has a representative which is obtained this way.

Therefore, listing all the possible ways of choosing the $3 g+r+s-3$ pairs and eliminating the choices giving rise to $\mathscr{M}_{g, r}^{s}$-equivalent pants decompositions, we get a representative for the $\mathscr{M}_{g, r}^{s}$-orbit of each maximal simplex; 


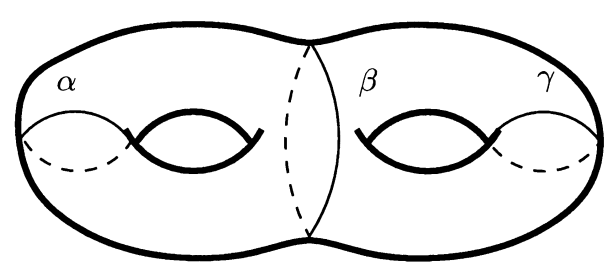

Figure 4.4. $G$-equivalence in $X$ vs $G$-equivalence in $X^{\text {ord }}$.

(2) Then, to get the representatives for the $\mathscr{M}_{g, r}^{s}$-orbits of the $k$-simplices of $X$, we just consider all the subfamilies of $k+1$ elements of every representative of a $\mathscr{M}_{g, r}^{s}$-orbit of pants decompositions, and we eliminate the choices giving rise to $\mathscr{M}_{g, r}^{s}$-equivalent families.

This shows that the number of pants decompositions (up to $\mathscr{M}_{g, r}^{s}$-equivalence) is finite; in particular, the 2-skeleton of $\bar{X}$ is also finite.

If we consider $X^{\text {ord }}$ instead of $X$, the only difference introduced by the ordering is that we do not allow permutations as in Proposition 9 above: for example, in Figure 4.4 the two families $\{\alpha, \beta\}$ and $\{\beta, \gamma\}$, though $\mathscr{M}_{g, r}^{s}$-equivalent as edges of $X$, are not $\mathscr{M}_{g, r}^{s}$-equivalent as edges of $X^{\text {ord }}$. Hence, the finiteness of the 2-skeleton of the quotient, required in Theorem 3, is preserved.

4.2 The presentation of the stabilizers. Consider an exact sequence of groups

$$
0 \rightarrow K \stackrel{i}{\rightarrow} G \stackrel{p}{\rightarrow} H \rightarrow 0
$$

and assume that $K$ (that we identify with $i(K)$ in $G)$ and $H$ have the presentations

$$
K=\left\langle G_{K} \mid R_{K}\right\rangle \text { and } H=\left\langle G_{H} \mid R_{H}\right\rangle .
$$

For each $y \in G_{H}$ we choose an element $\tilde{y} \in G$ such that $p(\tilde{y})=y$, and for each relation $r=y_{1} \ldots y_{m} \in R_{H}$ we set $\tilde{r}=\tilde{y}_{1} \ldots \tilde{y}_{m} \in G$; as the sequence is exact, for every $r \in R_{H}$ there exists a word $w_{r}$ in the elements of $G_{K}$ such that $\tilde{r}=w_{r}$ in $G$. Moreover, for each $x \in G_{K}$ and for each $y \in G_{H}$ there exists a word $g_{x, y} \in G$ such that $\tilde{y} x \tilde{y}^{-1}=g_{x, y}$ in $G$.

We omit the simple proof of the following Lemma:

Lemma 10. With the notation as above, $G$ admits the presentation

$$
G=\left\langle G_{K} \cup \bigcup_{y \in G_{H}} \tilde{y} \mid R^{(1)} \cup R^{(2)} \cup R^{(3)}\right\rangle
$$

where

$$
\begin{aligned}
& R^{(1)}=R_{K} ; \\
& R^{(2)}=\left\{\tilde{r} w_{r}^{-1} \mid r \in R_{H}\right\} ; \\
& R^{(3)}=\left\{\tilde{y} x \tilde{y}^{-1} g_{x, y}^{-1} \mid x \in G_{K}, y \in G_{H}\right\} .
\end{aligned}
$$


In order to get a presentation for the isotropy subgroup of the simplices of $X^{\text {ord }}$, we consider the following exact sequences:

$$
\begin{aligned}
& 0 \rightarrow \operatorname{Stab}^{+}(\alpha) \stackrel{i_{1}}{\rightarrow} \operatorname{Stab}(\alpha) \stackrel{p_{1}}{\rightarrow} H_{\alpha} \rightarrow 0 \\
& 0 \rightarrow \mathbb{Z}^{n} \stackrel{i_{2}}{\rightarrow} \mathscr{M}\left(F_{\alpha}\right) \stackrel{p_{2}}{\rightarrow} \operatorname{Stab}^{+}(\alpha) \rightarrow 0
\end{aligned}
$$

where:

$\alpha \quad$ is an $(n-1)$-simplex $\left\langle\alpha_{1}, \ldots, \alpha_{n}\right\rangle$ of $X^{\text {ord }}$;

$\operatorname{Stab}^{+}(\alpha)$ is the subgroup of $\operatorname{Stab}(\alpha)$ containing the elements leaving invariant each element of $\alpha$ with its orientation;

$H_{\alpha} \quad$ is the image of $p_{1}$ in the group $L(n)$, which is the group of the linear transformations $f \in \mathrm{GL}\left(\mathbb{R}^{n}\right)$ such that $f\left(e_{i}\right)= \pm e_{i}$ for each $e_{i}$, where $\left\{e_{1}, \ldots, e_{n}\right\}$ is the canonical basis of $\mathbb{R}^{n}$;

$p_{1} \quad$ is the natural homomorphism from $\operatorname{Stab}(\alpha)$ to $L(n)$ defined as follows: let $g$ be an element of $\operatorname{Stab}(\alpha)$, and let $h \in \mathscr{H}(F, P)$ representing $g$; we set

$$
p_{1}(g)\left[e_{i}\right]= \begin{cases}e_{i} & \text { if } h\left(\alpha_{i}\right)=\alpha_{i}, \\ -e_{i} & \text { if } h\left(\alpha_{i}\right)=\alpha_{i}^{-1}\end{cases}
$$

$\mathscr{M}\left(F_{\alpha}\right) \quad$ is the mapping class group of the surface $F_{\alpha}$.

Let us show the exactness of the two sequences: as far as (4.1) is concerned, we just remark that by an easy analysis case by case it is possible to describe $H_{\alpha}$ exactly (it is sufficient to consider the orientation preserving homeomorphisms of $F$, fixing the support of each component of $\alpha$, and possibly changing the orientation of some component).

Regarding (4.2), first of all we remark that, if $N_{1}, \ldots, N_{r}$ are the connected components of $F_{\alpha}$, we have

$$
\mathscr{M}\left(F_{\alpha}\right)=\mathscr{M}\left(N_{1}\right) \times \cdots \times \mathscr{M}\left(N_{r}\right) .
$$

Then we recall the map $\rho_{\alpha}: F_{\alpha} \rightarrow F$, which induces a homomorphism of groups $\left(\rho_{\alpha}\right)_{*}: \mathscr{M}\left(F_{\alpha}\right) \rightarrow \mathscr{M}(F)$, whose image is exactly $\mathrm{Stab}^{+}(\alpha)$ (in other words, $p_{2}$ is $\left.\left(\rho_{\alpha}\right)_{*}\right)$. Since it is possible to prove (see [29]) that, if $\gamma_{i}$ and $\gamma_{i}^{\prime}$ are the boundary curves of $F_{\alpha}$ such that $\rho_{\alpha} \circ \gamma_{i}=\rho_{\alpha} \circ \gamma_{i}^{\prime}=\alpha_{i}$, then ker $p_{2}$ is generated by $\left\{C_{1}\left(C_{1}^{\prime}\right)^{-1}, \ldots, C_{n}\left(C_{n}^{\prime}\right)^{-1}\right\}$ and it is a free Abelian group of rank $n$, sequence (4.2) is exact.

Using (4.2), we find a presentation for $\mathrm{Stab}^{+}(\alpha)$; then, applying Lemma 10 to (4.1), we get a presentation for the isotropy subgroup of $\alpha$.

Remark 11. Actually we are interested only in the presentations for the isotropy subgroups of the vertices and 1-simplices. Moreover, we will see in Section 6 that in the non-punctured case, the presentations for the vertices are enough.

As an example, and since in Section 7 we will use explicitly the presentations of the isotropy subgroups of the vertices, we describe them in detail. We say that a 
vertex $v$ of $\bar{X}^{\text {ord }}$ is non-separating if $F_{s(v)}$ is connected, where $s(v)$ is any representative for $v$ in $F$, and we call $v$ separating otherwise. Notice that there exists only one nonseparating vertex, that we will denote by $v_{0}$, and for such a vertex $F_{s\left(v_{0}\right)}$ is homeomorphic to $F_{g-1, r+2}^{s}$, while there are as many separating vertices as the number of different ways to divide $F$ into two connected components (up to homeomorphisms fixing the boundary components of $F$ ). Moreover, notice that $H_{s\left(v_{0}\right)}$ is always $\mathbb{Z}_{2}$, because there exists an element of $\operatorname{Stab} s\left(v_{0}\right)$ reversing the orientation of the nonseparating curve $s\left(v_{0}\right)$. On the contrary, for the separating vertices $H_{s(v)}$ is 0 except in one case, when $(g, r, s)=(2 k, 0,2 h)$ and $v$ is such that $F_{s(v)}$ has two connected components, both homeomorphic to $F_{k, 1}^{h}$. In this case, we denote such a vertex by $v_{\text {sym }}$, referring to it as the symmetric separating vertex, and, since there exists an element of $\operatorname{Stab} s\left(v_{\mathrm{sym}}\right)$ reversing the orientation of $s\left(v_{\mathrm{sym}}\right)$, we conclude that $H_{s\left(v_{\mathrm{sym}}\right)}$ is $\mathbb{Z}_{2}$.

Hence, if $\alpha$ is $s\left(v_{0}\right),(4.1)$ and (4.2) become

$$
\begin{aligned}
& 0 \rightarrow \operatorname{Stab}^{+}\left(s\left(v_{0}\right)\right) \rightarrow \operatorname{Stab}\left(s\left(v_{0}\right)\right) \rightarrow \mathbb{Z}_{2} \rightarrow 0, \\
& 0 \rightarrow \mathbb{Z} \rightarrow \mathscr{M}\left(F_{g-1, r+2}^{s}\right) \rightarrow \operatorname{Stab}^{+}\left(s\left(v_{0}\right)\right) \rightarrow 0,
\end{aligned}
$$

while if $\alpha$ is $s\left(v_{\text {sym }}\right)$ they become

$$
\begin{aligned}
& 0 \rightarrow \operatorname{Stab}^{+}\left(s\left(v_{\text {sym }}\right)\right) \rightarrow \operatorname{Stab}\left(s\left(v_{\text {sym }}\right)\right) \rightarrow \mathbb{Z}_{2} \rightarrow 0, \\
& 0 \rightarrow \mathbb{Z} \rightarrow \mathscr{M}\left(F_{k, 1}^{h}\right) \times \mathscr{M}\left(F_{k, 1}^{h}\right) \rightarrow \operatorname{Stab}^{+}\left(s\left(v_{\mathrm{sym}}\right)\right) \rightarrow 0 .
\end{aligned}
$$

Finally, if $\alpha$ is a representative for any separating and non-symmetric vertex, we have the exact sequence

$$
0 \rightarrow \mathbb{Z} \rightarrow \mathscr{M}\left(F_{g^{\prime}, r^{\prime}}^{s^{\prime}}\right) \times \mathscr{M}\left(F_{g-g^{\prime}, r-r^{\prime}+2}^{s-s^{\prime}}\right) \rightarrow \operatorname{Stab}(s(v)) \rightarrow 0 .
$$

Thus, if we know the presentations

$$
\begin{gathered}
\mathscr{M}\left(F_{g-1, r+2}^{s}\right)=\left\langle G_{0} \mid R_{0}\right\rangle, \\
\mathscr{M}\left(F_{k, 1}^{h}\right)=\left\langle G_{1} \mid R_{1}\right\rangle, \\
\mathscr{M}\left(F_{g^{\prime}, r^{\prime}}^{s^{\prime}}\right)=\left\langle G_{2} \mid R_{2}\right\rangle, \\
\mathscr{M}\left(F_{g-g^{\prime}, r-r^{\prime}+2}^{s-s^{\prime}}\right)=\left\langle G_{3} \mid R_{3}\right\rangle,
\end{gathered}
$$

Lemma 10 allows us to conclude that

$$
\begin{gathered}
\operatorname{Stab} s\left(v_{0}\right)=\left\langle G_{0} \cup \psi \mid R_{0} \cup R_{g} \cup\left\{\psi^{2} w_{\psi^{2}}\right\} \cup R_{m}\right\rangle, \\
\operatorname{Stab} s\left(v_{\mathrm{sym}}\right)=\left\langle G_{1} \cup \widetilde{G_{1}} \cup \rho \mid R_{1} \cup \widetilde{R_{1}} \cup R_{c} \cup R_{g} \cup\left\{\rho^{2} w_{\rho^{2}}\right\} \cup R_{m}\right\rangle, \\
\operatorname{Stab} s(v)=\left\langle G_{2} \cup G_{3} \mid R_{2} \cup R_{3} \cup R_{c} \cup R_{g}\right\rangle,
\end{gathered}
$$



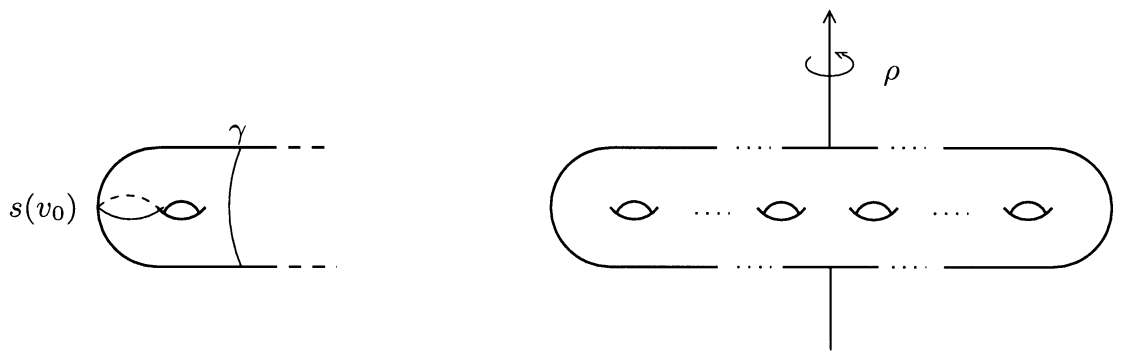

Figure 4.5. The curves $s\left(v_{0}\right)$ and $\gamma$ and the element $\rho$.

where we identify each $\mathrm{Stab}^{+}$with its image in $\mathrm{Stab}$, we denote by $\left\langle\widetilde{G_{1}} \mid \widetilde{R_{1}}\right\rangle$ the presentation of the second copy of $\mathscr{M}\left(F_{k, 1}^{h}\right)$ in $\mathscr{M}\left(F_{S\left(v_{\text {sym }}\right)}\right), w_{\psi^{2}}$ and $w_{\rho^{2}}$ are as in the notation of Lemma 10, and where

$\psi \quad$ is an element of $\operatorname{Stab} s\left(v_{0}\right)$ reversing the orientation of $s\left(v_{0}\right)$ (the $\tilde{1}$ corresponding to the generator 1 of $\mathbb{Z}_{2}$ in (4.3) with the notation of Lemma 10);

$\rho \quad$ is an element of $\operatorname{Stab} s\left(v_{\mathrm{sym}}\right)$ reversing the orientation of $s\left(v_{\mathrm{sym}}\right)$ (the $\tilde{1}$ corresponding to the generator 1 of $\mathbb{Z}_{2}$ in (4.4));

$R_{g} \quad$ is the relation identifying the Dehn twists along the two boundary components of $F_{s(v)}$ corresponding to the curve $s(v)$;

$R_{m}$ are the relations of type $R^{(3)}$ with the notation of the Lemma 10, "mixing", $\mathrm{Stab}^{+}$with $\mathbb{Z}_{2}$;

$R_{c} \quad$ are the relations saying that the generators of the mapping class group of one of the connected components of $F_{S(v)}$ commute with those of the other in $\mathscr{M}\left(F_{S(v)}\right)$.

It is always possible to choose for $\psi$ the semitwist along c relative to $s\left(v_{0}\right)$ (see the appendix for its definition), and for $\rho$ the rotation of $\pi$ around the $z$ axis (see Figure 4.5). Hence, the relation $\rho^{2} w_{\rho^{2}}$ becomes $\rho^{2}$ (i.e. $\rho^{2}=1$ ), while it is not difficult to prove that $\psi^{2}$ is the Dehn twist along the curve $\gamma$, and thus to find the expression for $w_{\psi}$.

\section{The inductive process and the sporadic surfaces}

The general method presented in Section 2 applies to the ordered complex of curves of any surface $F_{g, r}^{s}$, provided this complex is simply connected, and provided we have a finite presentation for the isotropy subgroups of a representative in every $\mathscr{M}_{g, r^{-}}^{s}$ equivalence class of its vertices and edges.

Subsection 4.2 describes how to produce a presentation for the isotropy subgroup of any generic ordered $k$-family of closed curves $\alpha$, if a presentation is known for the mapping class group of the surface obtained by $F_{g, r}^{s}$ cutting it open along $\alpha$. Moreover, if such a presentation is finite, the same is true for the resulting one of the isotropy subgroup.

Cutting open $F_{g, r}^{s}$ along a representative for every vertex and every edge, we find subsurfaces $F_{g^{\prime}, r^{\prime}}^{s^{\prime}}$ with $\left(g^{\prime}, r^{\prime}+s^{\prime \prime}\right)<(g, r+s)$ (with the lexicographic ordering), and when these surfaces are not sporadic we can apply again the previous argument to compute a presentation for their mapping class group. 

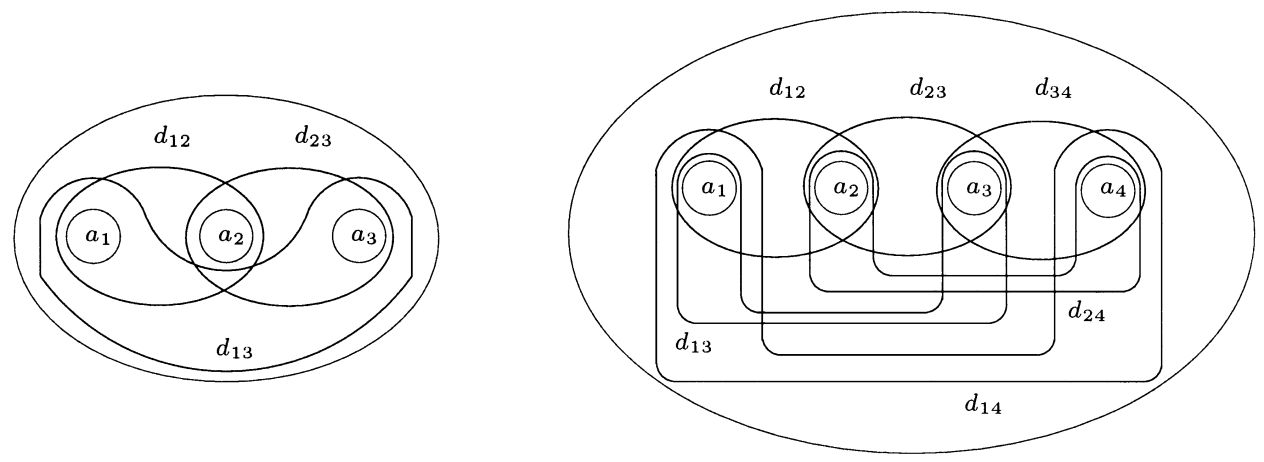

Figure 5.1. Generators for the mapping class group of $F_{0,4}^{0}$ and $F_{0,5}^{0}$.

Repeating recursively these steps for any non-sporadic $F_{g^{\prime}, r^{\prime}}^{s^{\prime}}$, we finally arrive at sporadic surfaces: hence if we know a finite presentation for the mapping class group of each sporadic surface (as a basis of this inductive process), we inductively get a finite presentation for $\mathscr{M}_{g, r}^{s}$.

The sporadic surfaces $F_{g^{\prime}, r^{\prime}}^{s^{\prime}}$ which may appear in this process are the sphere with $r^{\prime}+s^{\prime}=3,4,5$ and the torus with $r^{\prime}+s^{\prime}=1,2$ (by Definition 7). All these cases are well known: $\mathscr{M}\left(F_{0,3}^{0}\right)=\mathbb{Z}^{3}$, generated by the Dehn twists along the boundary components, and in general it is a classical result (see for example $[1,11]$ or [8]), that, for $r \geqslant 1, \mathscr{M}\left(F_{0, r}^{0}\right)=P_{r-1} \times \mathbb{Z}^{r-1}$, where $P_{n}$ is the group of pure braids with $n$ strings. In particular, in the cases we are concerned with,

- $\mathscr{M}\left(F_{0,4}^{0}\right)=P_{3} \times \mathbb{Z}^{3}$, and it admits a presentation with

Generators: $\left\{A_{1}, A_{2}, A_{3}, D_{12}, D_{13}, D_{23}\right\}$ where the curves $a_{i}, d_{i j}$ are represented in

Figure 5.1 (we recall that, if not otherwise stated, we denote a curve by a lowercase letter, and the Dehn twist along the same curve by the corresponding capital letter).

Relations: $A_{i} A_{j}=A_{j} A_{i}$ for all $i, j$

$A_{i} D_{j k}=D_{j k} A_{i}$ for all $i, j, k$

$D_{23} D_{13} D_{12}=D_{13} D_{12} D_{23}$

$D_{23} D_{13} D_{12}=D_{12} D_{23} D_{13}$

- $\mathscr{M}\left(F_{0,5}^{0}\right)=P_{4} \times \mathbb{Z}^{4}$, and it admits a presentation with

Generators: $\left\{A_{1}, A_{2}, A_{3}, A_{4}, D_{12}, D_{13}, D_{14}, D_{23}, D_{24}, D_{34}\right\}$ where the curves $a_{i}, d_{i j}$ are represented in Figure 5.1.

$\begin{array}{ll}\text { Relations: } & A_{i} A_{j}=A_{j} A_{i} \quad \text { for all } i, j \\ & A_{i} D_{j k}=D_{j k} A_{i} \quad \text { for all } i, j, k \\ & D_{34} D_{12}=D_{12} D_{34} \\ & D_{14} D_{23}=D_{23} D_{14} \\ & D_{23} D_{13} D_{12}=D_{13} D_{12} D_{23}=D_{12} D_{23} D_{13} \\ & D_{24} D_{14} D_{12}=D_{14} D_{12} D_{24}=D_{12} D_{24} D_{14} \\ & D_{34} D_{14} D_{13}=D_{14} D_{13} D_{34}=D_{13} D_{34} D_{14} \\ & D_{34} D_{24} D_{23}=D_{24} D_{23} D_{34}=D_{23} D_{34} D_{24} \\ & D_{24} D_{34}^{-1} D_{14}^{-1} D_{34} D_{14} D_{13}=D_{34}^{-1} D_{14}^{-1} D_{34} D_{14} D_{13} D_{24}\end{array}$ 
$a$
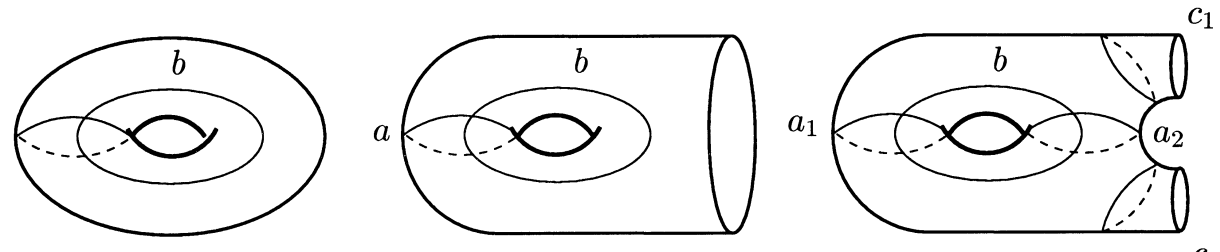

Figure 5.2. Generators for the mapping class group of $F_{1, r}^{0}, r=0,1,2$.

In what follows, we will refer to these presentations as standard presentations for the sporadic surfaces of genus 0 .

As far as the sporadic surfaces of genus 1 are concerned,

- $\mathscr{M}\left(F_{1,0}^{0}\right)=\langle A, B \mid A B A=B A B\rangle=S L(2, \mathbb{Z})(a, b$ as in Figure 5.2);

- $\mathscr{M}\left(F_{1,1}^{0}\right)=S L(2, \mathbb{Z})$, and admits the presentation $\mathscr{M}\left(F_{1,1}^{0}\right)=\langle A, B \mid A B A=B A B\rangle$ $(a, b$ as in Figure 5.2);

- for $\mathscr{M}\left(F_{1,2}^{0}\right)$ we have, with $a_{1}, a_{2}, b, c_{1}, c_{2}$ as in Figure 5.2, the following presentation:

Generators: $\quad\left\{A_{1}, A_{2}, B, C_{1}, C_{2}\right\}$

Relations: $\quad A_{1} A_{2}=A_{2} A_{1}$

$$
\begin{array}{ll}
A_{i} B A_{i}=B A_{i} B & i=1,2 \\
A_{i} C_{j}=C_{j} A_{i} & i, j=1,2 \\
C_{j} B=B C_{j} & j=1,2 \\
\left(A_{1}^{2} A_{2} B\right)^{3}=C_{1} C_{2} & \\
\left(A_{1} A_{2}^{2} B\right)^{3}=C_{2} C_{1} &
\end{array}
$$

These presentations are particular cases of a general result of Gervais in [7] (see Section 7); therefore in what follows we will refer to them as to Gervais presentations for the sporadic surfaces of genus 1 .

Since we will discuss in detail only the non-punctured case in the next sections, we avoid to describe here the mapping class group of the sporadic surfaces with punctures. We just remark that at least one simple finite presentation for each of them is well known, so our inductive argument works also in the punctured case. We refer the interested reader to $[1,6]$ for the case of genus 0 , and to [20] for the case of genus 1 .

\section{The non-punctured case}

Proposition 12. If $F_{g, r}=F_{g, r}^{0}$ is not sporadic, and $g>0$, then for every choice of the representatives and of the maximal tree, it is possible to express all the symbols $E$ appearing in Theorem 3 as a product of elements in $\bigcup_{v \in V\left(\bar{X}^{\text {ord }}\right)} \operatorname{Stab} s(v)$. Hence, the presentation obtained applying Theorem 3 to the action of the mapping class group on the ordered complex of curves reduces to

$$
\mathscr{M}\left(F_{g, r}\right)=\left\langle\bigcup_{v \in V\left(\bar{X}^{\text {ord }}\right)} S_{v} \mid \bigcup_{v \in V\left(\bar{X}^{\text {ord }}\right)} R_{v} \cup \widetilde{R^{(3)}} \cup \widetilde{R^{(4)}}\right\rangle,
$$


where the $\widetilde{R^{(n)}}$ are the relations obtained substituting in the $R^{(n)}$ the expressions for the symbols $E$ corresponding to the edges.

Proof. The relation of type $R^{(4)}$ carried by a triangle $T$ with edges $a, b, c$ is

$$
\gamma_{c, i} \gamma_{T, c} \gamma_{T, a}^{-1} \gamma_{a, i}^{-1} A \gamma_{a, t} \gamma_{T, a} \gamma_{T, b}^{-1} \gamma_{b, i}^{-1} B \gamma_{b, t} \gamma_{T, b} \gamma_{T, c}^{-1} \gamma_{c, t}^{-1}
$$

where

$$
\begin{aligned}
& \gamma_{c, i} \gamma_{T, c} \gamma_{T, a}^{-1} \gamma_{a, i}^{-1} \in \operatorname{Stab} s(f), \\
& \gamma_{a, t} \gamma_{T, a} \gamma_{T, b}^{-1} \gamma_{b, i}^{-1} \in \operatorname{Stab} s(m), \\
& \gamma_{b, t} \gamma_{T, b} \gamma_{T, c}^{-1} \gamma_{c, t}^{-1} \in \operatorname{Stab} s(p) .
\end{aligned}
$$

Therefore, it two of the edges of $T$ are in the maximal tree $\mathscr{T}$, once we "kill" $\mathscr{T}$ using the relations $R^{(2)}$, the relation (6.2) gives an expression for the third edge as a product of stabilizers of the representatives for the vertices. The same is true if in (6.2) two of the symbols for the edges were already expressed as products of stabilizers.

We say that a symbol $E$ is determinable (or simply that the corresponding edge $e$ is determinable) if, using recursively relations of type $R^{(4)}$, after "killing" the maximal tree it is possible to express $E$ as a product of elements in $\bigcup_{v \in V\left(\bar{X}^{\text {ord }}\right)} S_{v}$.

Given an edge $e$ in $\bar{X}^{\text {ord }}$, we call topological inverse of $e$ the edge $\bar{e}$ being the same element in $\bar{X}$, but having the opposite orientation, i.e. directed from $t(e)$ to $i(e)$. It is clear that, if there exists in $\bar{X}^{\text {ord }}$ a 2-cell with edges $a, b, c$, then there are also all the 2-cells with the same vertices that one can build using $a, b, c$ and their topological inverses (see Figure 3.2). Therefore, if two of the edges $a, b, c$ are determinable, so is the third one and the three topological inverses as well.

Since $g>0$, there exists a single non-separating vertex in $\bar{X}^{\text {ord }}$ that we denote by $v_{0}$.

Lemma 13. For each $v \in V(\bar{X}), v \neq v_{0}$, there always exists at least one edge $e$ with $i(e)=v_{0}$ and $t(e)=v$. More precisely, the number of such edges is 1 if one of the connected components of $F_{v}$ has genus 0 , or if $v=v_{\mathrm{sym}}$, and it is 2 otherwise.

Proof. The assertion follows immediately from the analysis of Subsection 4.1.

Lemma 14. For each $v \in V(\bar{X}), v \neq v_{0}$, at least one of the edges joining $v_{0}$ and $v$ is determinable.

Proof. Let $e \in \mathscr{T}$ be an edge joining $v_{0}$ and a separating vertex, say $v_{1}$. Then (at least) one among $v_{0}$ and $v_{1}$ is connected with another vertex $v_{2}$ by an edge $e^{\prime}$ belonging to the maximal tree $\mathscr{T}$. If $e^{\prime}$ joins the two separating vertices, then there always exists a 2-cell with vertices $v_{0}, v_{1}, v_{2}$, containing the two edges $e$ and $e^{\prime}$ : this follows from the fact that any representative for $e^{\prime}$ divides $F$ into three subsurfaces, and at least one of them has genus $>0$. Therefore we may always find a representative for the non-separating vertex disjoint from the other two chosen representatives (that means we always have a triangle $T$ with vertices $v_{0}, v_{1}$ and $v_{2}$ ); moreover, if there is only one 
component of genus $>0$, we are sure we are realizing the edge $e$, because there is only one edge joining $v_{0}$ and $v_{1}$. If, on the contrary, the connected components with genus $>0$ are more than one, we may always choose where to put the representative for $v_{0}$ in order to get a representative for $e$. Hence, in any case, as two of the edges of the 2-cell $T$ are in $\mathscr{T}$, the third one is determinable (and the same is true for the topological inverses).

Thus we have proven the assertion for $v_{1}$ and $v_{2}$, and the same is trivially true if the edge $e^{\prime}$ joins $v_{0}$ and $v_{2}$. Applying recursively the same argument (as we move along the maximal tree touching all the other vertices), we get the assertion for every $v \in V(\bar{X})$.

In the sequel, we sometimes write $e_{i, j}^{(n)}$ to denote an edge in $\bar{X}^{\text {ord }}$ with initial vertex $v_{i}$ and terminal vertex $v_{j}$, emphasizing, by means of the progressive number $n$, the fact that in general such an edge is not unique.

Lemma 15. Let $e_{0,0}^{(0)}$ be the loop of $\bar{X}^{\text {ord }}$ based in $v_{0}$ and such that $F_{s\left(e_{0,0}^{(0)}\right)}$ is connected (and therefore homeomorphic to $\left.F_{g-2, r+4}\right)$; then $e_{0,0}^{(0)}$ is determinable.

Proof. Let us suppose there exists an edge $e \in \mathscr{T}$ with one end in $v_{0}$ and such that there exists a 2-cell with edges $e_{0,0}^{(0)}, e, e$. In such a situation, the symbol corresponding to $e_{0,0}^{(0)}$ is determinable by the relation $R^{(4)}$ corresponding to that 2 -cell.

If, on the contrary, such an edge does not exist, this means that $g \geqslant 2$, and the only $e \in \mathscr{T}$ with one end in $v_{0}$ is the edge such that $F_{S(e)}$ has a connected component homeomorphic to the pair of pants $F_{0,3}$ and the other one to $F_{g-1, r+1}$, with $(g-1, r+1)>(1,1)$. In this case, the other end of $e$, that we denote by $v_{1}$, is connected to another vertex $v_{2}$ by an edge $e^{\prime} \in \mathscr{T}$, and there exists a triangle with vertices $v_{0}, v_{1}, v_{2}$ and edges $e, e^{\prime}$ and $e^{\prime \prime}$, where $e^{\prime \prime}$ is an edge with ends $v_{0}$ and $v_{2}$. Therefore the edge $e^{\prime \prime}$ is determinable, and, since there exists a 2-cell with edges $e_{0,0}^{(0)}, e^{\prime \prime}, e^{\prime \prime}$, so is the loop $e_{0,0}^{(0)}$.

Lemma 16. The following edges are determinable:

(i) all the loops based in $v_{0}$;

(ii) all the edges with one end in $v_{0}$;

(iii) all the loops based in $v_{i}$, for every $i$;

(iv) all the edges $e_{i, j}^{(n)}$ with ends in $v_{i}$ and $v_{j}, i \neq j$ and $i, j \neq 0$.

Proof. (i) Given any loop $e_{0,0}^{(i)}$ based in $v_{0}$, different from $e_{0,0}^{(0)}$, if $g \geqslant 2$ there always exists a triangle with edges $e_{0,0}^{(i)}, e_{0,0}^{(0)}, e_{0,0}^{(0)}$, and then $e_{0,0}^{(i)}$ is determinable. In case $g=1$, we observe that there always exists a triangle with edges $e_{0,0}^{(i)}, e, e$, a representative of which is depicted in the left-hand side of Figure 6.1; if $e \in \mathscr{T}$, we are done, if not, then the two edges $e^{\prime}, e^{\prime \prime}$, whose representatives are shown again in Figure 6.1, are in $\mathscr{T}$. If this is the case, $e$ is determinable by the triangle $e, e^{\prime}, e^{\prime \prime}$ (right-hand side of Figure 6.1), and therefore the loop $e_{0,0}^{(i)}$ is determinable. 

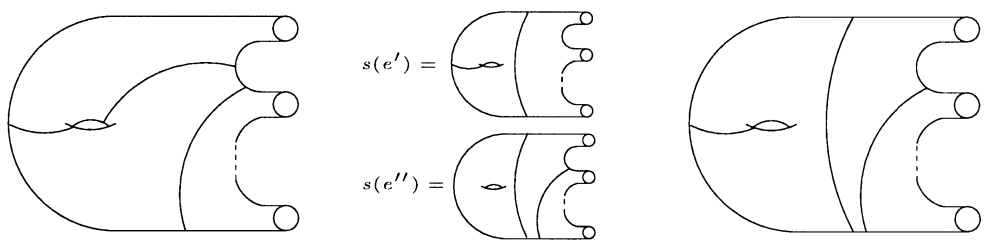

Figure 6.1. Determinability of a loop $e_{0,0}^{(i)}$ in $F_{1, r}$.

(ii) The assertion follows immediately from Lemma 14, Lemma 15, and the observation that, if $v$ is such that there are two different edges with initial vertex $v_{0}$ and terminal vertex $v$, then there exists a triangle whose edges are these two edges and the loop $e_{0,0}^{(0)}$.

(iii) Given any loop based in $v$, there always exists a triangle whose edges are this loop and two edges with one end in $v$ and the other one in $v_{0}$, hence we have the assertion.

(iv) Just notice that there always exists a triangle with edges $e_{i, j}^{(n)}$ and two edges with one end in $v_{0}$ and the other one respectively in $v_{i}$ and $v_{j}$.

This concludes the proof of Proposition 12.

Moreover, we have the following result:

Theorem 17. There exists a choice of the representatives and of the maximal tree such that (6.1) expresses $\mathscr{M}_{g, r}$ as the free product of the isotropy subgroups of the chosen representatives for the vertices, amalgamated along the subgroups $\operatorname{Stab} s(e)$, as e varies in $E\left(\bar{X}_{g, r}\right)$.

Proof. Let us fix a representative $s\left(v_{0}\right)$ for the non-separating vertex. It is always possible to choose, for every separating vertex $v_{i}$, a representative $s\left(v_{i}\right)$ such that $s\left(v_{0}\right) \cap s\left(v_{i}\right)=\varnothing$.

Hence, we may take $\mathscr{T}$ to be the union, for all the separating vertices $v_{i} \in V\left(\bar{X}_{g, r}\right)$, of the edges $e$ with $i(e)=v_{0}$ and $t(e)=v_{i}$, such that it is possible to choose $s(e)=$ $\left\langle s\left(v_{0}\right), s\left(v_{i}\right)\right\rangle$.

Concerning all the other edges, notice that we may always choose the representatives $s(e)$ in such a way that at least one of the ends of $s(e)$ is the chosen representative for its class, and we may always choose for the topological inverse $\bar{e}$ of the edge $e$ the representative $s(\bar{e})=\langle t(s(e)), i(s(e))\rangle$, so that $\gamma_{\bar{e}, i}=\gamma_{e, t}$ and $\gamma_{\bar{e}, t}=\gamma_{e, i}$.

By Proposition 12, every edge $e \notin \mathscr{T}$ is determinable; with the choices we made, it turns out that actually the symbol $E$ associated to an edge $e$ is determined as

$$
\begin{array}{ll}
E=1 & \text { if } i(s(e))=s(i(e)) \text { and } t(s(e))=s(t(e)), \\
E=\gamma_{e, t}^{-1} & \text { if } i(s(e))=s(i(e)) \text { and } t(s(e)) \neq s(t(e)), \\
E=\gamma_{e, i} & \text { if } i(s(e)) \neq s(i(e)) \text { and } t(s(e))=s(t(e)),
\end{array}
$$

where the $\gamma_{e, i}$ and $\gamma_{e, t}$ may be taken to be products of stabilizers of suitable vertices. 
Hence, it is obvious that all the relations of type $R^{(4)}$ are coherent, i.e. they vanish once we substitute the expressions $(6.3)$.

Moreover, for each edge $e \in E\left(\bar{X}_{g, r}^{\text {ord }}\right)$ determined as $E=1$, it is immediate that the corresponding relation of type $R^{(3)}$ identifies the copy of $\operatorname{Stab} s(t(e)) \cap \operatorname{Stab} s(i(e))$ (that is $\operatorname{Stab} s(e))$ in $\operatorname{Stab} s(t(e))$ with the copy of the same subgroup in $\operatorname{Stab} s(i(e))$ (and the relation corresponding to the topological inverse also). The relations of type $R^{(3)}$ corresponding to the other edges identify the copy of Stab $t(s(e)) \cap \operatorname{Stab} i(s(e))$ in $\operatorname{Stab} t(s(e))$ with the copy of the same subgroup in Stab $i(s(e))$, after, by means of suitable conjugations, expressing the elements of the intersection as products of elements in $\bigcup_{v \in V\left(\bar{X}_{g, r}\right)} \operatorname{Stab} s(v)$.

The proofs we gave of Proposition 12 and Theorem 17 strongly depend on the existence of a non-separating vertex, $v_{0}$; hence, the argument obviously does not work for the case of genus 0 .

Actually, the structure of $\bar{X}_{0, r}$ is quite different from that of $\bar{X}_{g, r}$ when $g \geqslant 1$; in order to describe it, we enumerate the boundary components of $F_{0, r}$, denoting them by $\partial_{1}, \partial_{2}, \ldots, \partial_{r}$.

The vertices of $\bar{X}_{0, r}$ are $v_{I}$, with multi-index $I=\left(i_{1}, i_{2}, \ldots, i_{s}\right)$ such that

$1 \leqslant i_{1}<i_{2}<\cdots<i_{s} \leqslant r$

$s=2, \ldots,[r / 2]$ where $[x]$ denotes the integer part of $x$

(and, if $r$ is even, the $v_{I}$ with $\# I=r / 2$ have $i_{1}=1$ ),

where $v_{i_{1}, \ldots, i_{s}}$ denotes the $\mathscr{M}_{0, r}$-equivalence class of a curve $s\left(v_{i_{1}, \ldots, i_{s}}\right)$ separating $F_{0, r}$ into two connected components, both of genus 0 , such that one of them has boundary components $\partial_{i_{1}}, \ldots, \partial_{i_{s}}$ and $s\left(v_{i_{1}, \ldots, i_{s}}\right)$.

Regarding the edges of $\bar{X}_{0, r}$, it is easy to verify the following claims:

Lemma 18. (i) There are no loops;

(ii) for each pair of vertices $v_{I}$ and $v_{J}$ there exists at most one edge with initial vertex $v_{I}$ and terminal vertex $v_{J}$;

(iii) there exists an edge connecting $v_{I}$ and $v_{J}$ if and only if $4 \leqslant \# I+\# J \leqslant r-1$ and, if $\# I \leqslant \# J$, either $I \subsetneq J$ or $I \cap J=\varnothing$. If such an edge exists, we denote it by $e_{I, J}$.

Finally, the triangles of $\bar{X}_{0, r}$ are the $T_{I, J, K}=\left[v_{I}, v_{J}, v_{K}\right]$ such that $6 \leqslant \# I+\# J+\# K$ $\leqslant r$ and, if $\# I \leqslant \# J \leqslant \# K$, we have one of the following situations:

$\cdot I \cap J=\varnothing, \quad J \cap K=\varnothing, \quad I \cap K=\varnothing$;

- $J \cap K=\varnothing, \quad I \subsetneq K$;

- $I \cap K=\varnothing, \quad J \subsetneq K$;

- $I \cap J=\varnothing, \quad J \subsetneq K, \quad I \subsetneq K$;

- $I \subsetneq J \subsetneq K$. 

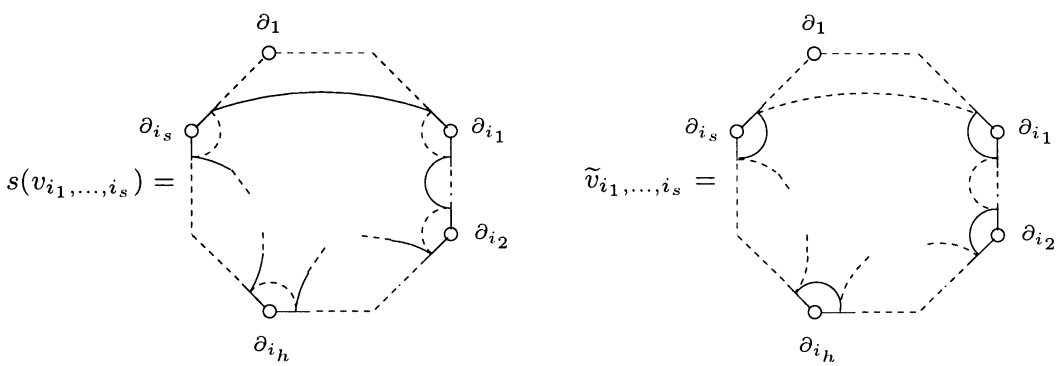

Figure 6.2. Representatives for the vertices of $\bar{X}_{0, r}$.

Let us choose representatives for the vertices as in Figure 6.2. Hence, it is possible to choose representatives for the edges such that $s\left(e_{I, J}\right)=\left\{s\left(v_{I}\right), s\left(v_{J}\right)\right\}$ if either $\# I<\# J$ and $I \subsetneq J$, or $I \cap J=\varnothing$ and the $i_{h}$ 's do not alternate with the $j_{k}$ 's with respect to the cyclic ordering of $\{1, \ldots, r\}$. In the other cases, we may choose $s\left(e_{I, J}\right)$ $=\left\{s\left(v_{I}\right), \tilde{v}_{J}\right\}$, where $I$ is the multi-index with greater cardinality, or, if $\# I=\# J, I$ contains the lower index (where $\tilde{v}_{J}$ is the representative for $v_{J}$ depicted in the righthand side of Figure 6.2).

We restrict from now on to the case of $F_{0,6}$, the first non-sporadic surface of genus 0 , that, in spite of its simplicity, is paradigmatic of the case $F_{0, r}$.

We choose the maximal tree $\mathscr{T}$ as follows: we put in $\mathscr{T}$ the ten edges having one end in $v_{1,2}$ :

$$
\begin{array}{lllll}
e_{(1,2),(3,4)}, & e_{(1,2),(3,6)}, & e_{(1,2),(4,6)}, & e_{(1,2),(1,2,3)}, & e_{(1,2),(1,2,5)} \\
e_{(1,2),(3,5)}, & e_{(1,2),(4,5)}, & e_{(1,2),(5,6)}, & e_{(1,2),(1,2,4)}, & e_{(1,2),(1,2,6)} .
\end{array}
$$

From what we said before, $v_{1,2}$ appears in 15 triangles, and clearly all of them have two edges in $\mathscr{T}$; hence, the 15 other edges are determined, more precisely they are:

$$
\begin{array}{lllll}
e_{(3,4),(5,6)}, & e_{(1,2,3),(4,5)}, & e_{(1,2,4),(3,5)}, & e_{(1,2,5),(3,4)}, & e_{(1,2,6),(3,4)}, \\
e_{(3,5),(4,6)}, & e_{(1,2,3),(4,6)}, & e_{(1,2,4),(3,6)}, & e_{(1,2,5),(3,6)}, & e_{(1,2,6),(3,5)}, \\
e_{(4,5),(3,6)}, & e_{(1,2,3),(5,6)}, & e_{(1,2,4),(5,6)}, & e_{(1,2,5),(4,6)}, & e_{(1,2,6),(4,5) .} .
\end{array}
$$

Each of these edges appears in two more triangles, whose third vertex is still not reached by the maximal tree: for example, $e_{(3,4),(5,6)}$ appears in $T_{(3,4),(5,6),(1,3,4)}$ and $T_{(3,4),(5,6),(1,5,6)}$. Hence, if we put in $\mathscr{T}$ the edges $e_{(1,3,4),(3,4)}$ and $e_{(1,5,6),(5,6)}$, we determine the edges $e_{(1,3,4),(5,6)}$ and $e_{(1,5,6),(3,4)}$. Applying the same argument to all the edges listed in (6.4), we complete the maximal tree adding the 14 edges

$$
\begin{array}{ll}
e_{(1, i, j),(i, j)} & 3 \leqslant i<j \leqslant 6 \\
e_{(1,2, j),(1, j)} & 3 \leqslant j \leqslant 6 \\
e_{(1,2, j),(2, j)} & 3 \leqslant j \leqslant 6
\end{array}
$$


and we determine the 30 edges

$$
\begin{array}{ll}
e_{(1, i, j),(k, l)} & \{i, j\} \cup\{k, l\}=\{3,4,5,6\}, \\
e_{(2, j),(k, l)} & j, k, l \in\{3,4,5,6\}, i \neq k, i \neq l, \\
e_{(1, j),(k, l)} & j, k, l \in\{3,4,5,6\}, i \neq k, i \neq l .
\end{array}
$$

Now, the argument applied again to each edge in (6.5) gives the remaining 36 edges. Moreover, it is easy to check that, independently from the choices of the representatives for the triangles, the expressions found for the symbols associated to the edges are:

$$
\begin{array}{ll}
E=1 & \text { if } i(s(e))=s(i(e)) \text { and } t(s(e))=s(t(e)), \\
E=\gamma_{e, i} & \text { if } i(s(e)) \neq s(i(e)) \text { and } t(s(e))=s(t(e)), \\
E=\gamma_{e, t}^{-1} & \text { if } i(s(e))=s(i(e)) \text { and } t(s(e)) \neq s(t(e)) .
\end{array}
$$

Hence, the relation of type $R^{(3)}$ associated to the edge $e$ identifies the copy of the intersection $\operatorname{Stab} t(s(e)) \cap \operatorname{Stab} i(s(e))$ (that is $\operatorname{Stab} s(e))$ in $\operatorname{Stab} t(s(e)$ ) with the copy of the same intersection in $\operatorname{Stab} i(s(e))$, while the relations of type $R^{(4)}$ that we did not use to determine the edges are "coherent", hence they disappear once we substitute the values obtained for the $E$ 's.

The generalization to the case $F_{0, r}, r>6$ is straightforward, which proves the following result, analogous to Theorem 17:

Theorem 19. There exists a choice of the representatives and of the maximal tree such that, for each non-sporadic surface of genus 0 , we may express $\mathscr{M}_{0, r}$ as the free product of the isotropy subgroups of the $s(v), v \in V\left(\bar{X}_{0, r}\right)$, amalgamated along the subgroups $\operatorname{Stab} s(e)$ with $e \in E\left(\bar{X}_{0, r}\right)$.

Remark 20. It is unknown to the author if the result of Theorem 19 is actually independent from the choice of the maximal tree.

\section{Recovering known presentations}

Let us consider, on the surface $F_{g, r}, g \geqslant 1, r \geqslant 0$, the curves of Figure 7.1. A triple $(i, j, k) \in\{1, \ldots, 2 g+r-2\}^{3}$ is said to be good when

$$
\begin{aligned}
& \text { i) }(i, j, k) \notin\{(l, l, l) \mid l \in\{1, \ldots, 2 g+r-2\}\} \text {, } \\
& \text { ii) } i \leqslant j \leqslant k \text { or } j \leqslant k \leqslant i \text { or } k \leqslant i \leqslant j .
\end{aligned}
$$

Recall that we denote each curve by a lowercase letter, and the Dehn twist along the same curve by the corresponding capital letter. Gervais, in [7], proved the following result: 

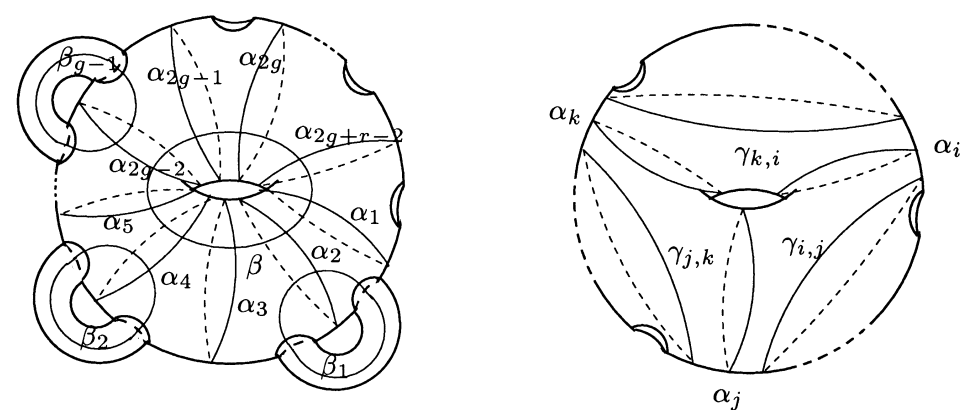

Figure 7.1. Gervais generators for $\mathscr{M}\left(F_{g, r}\right)$.

Theorem 21. For all $(g, r) \in \mathbb{N}^{*} \times \mathbb{N}$, the mapping class group $\mathscr{M}_{g, r}$ admits a presentation with

Generators: $\quad B, B_{1}, \ldots, B_{g-1}, A_{1}, \ldots, A_{2 g+r-2},\left(C_{i, j}\right)_{1 \leqslant i, j \leqslant 2 g+r-2, i \neq j}$

Relations: handles $C_{2 i, 2 i+1}=C_{2 i-1,2 i}$ for all $i, 1 \leqslant i \leqslant g-1$,

braids for all $X, Y$ among the generators, $X Y=Y X$ if the associated curves are disjoint and $X Y X=Y X Y$ if the associated curves intersect transversally in a single point,

stars $\quad C_{i, j} C_{j, k} C_{k, i}=\left(A_{i} A_{j} A_{k} B\right)^{3}$ for all good triples $(i, j, k)$, where we define $C_{l, l}=1$.

To prove this result, Gervais started from Wajnryb's presentation described in [31], which in turn was obtained exploiting the action of $\mathscr{M}(F)$ on the Hatcher-Thurston complex ([14]). We prove in this section that it is possible to get the Gervais presentation for the mapping class group of any non-sporadic surface $F_{g, r}$ using the ordered complex of curves in place of the Hatcher-Thurston complex, provided we take as starting point of the inductive process the Gervais presentation for the sporadic subsurfaces of genus 1, and the standard presentation for the sporadic subsurfaces of genus 0 (see Section 5).

Let us consider a non-sporadic surface $F_{g, r}$, of genus greater than 0 ; by Theorem 17 , its mapping class group is the free product of the isotropy subgroups of a (suitably chosen) representative for each class in $V\left(\bar{X}_{g, r}^{\text {ord }}\right)$, amalgamated along the subgroups $\operatorname{Stab} s(e)$, for $e \in E\left(\bar{X}^{\text {ord }}\right)$.

Hence, reasoning recursively, we just need to know some presentation for the sporadic surfaces $F_{0, r^{\prime}}, r^{\prime}=3,4,5$ and $F_{1, r^{\prime}}, r^{\prime}=1,2$.

Theorem 22. Starting from the Gervais presentation for $\mathscr{M}_{1,1}$ and $\mathscr{M}_{1,2}$, and the standard presentation for $\mathscr{M}_{0,3}, \mathscr{M}_{0,4}, \mathscr{M}_{0,5}$, we get the Gervais presentation for any nonsporadic $F_{g, r}$ of genus $g>0$.

Proof. The assertion follows immediately from the following lemma: 
Lemma 23. Let $F_{g, r}$ be a non-sporadic surface with $\left.g\right\rangle 0$. If $G_{v}=\left\langle S_{v} \mid R_{v}\right\rangle$ is a presentation for the isotropy group of a representative $s(v)$ of each vertex $v$ in $\bar{X}_{g, r}^{\text {ord }}$, computed starting from the Gervais presentation of the mapping class group for the connected components of $F_{S(v)}$ (or the standard presentation, in case one of this components has genus 0$)$, then the presentation of $\mathscr{M}\left(F_{g, r}\right)$ described in Theorem 17 is immediately reducible to the Gervais one.

Proof of the lemma. Let $\gamma_{1,2}$ be the chosen representative for the non-separating vertex $v_{0} \in V\left(\bar{X}_{g, r}^{\text {ord }}\right)$. Then, by (4.6), considering the Gervais presentation of $\mathscr{M}\left(F_{\gamma_{1,2}}\right)=$ $\mathscr{M}\left(F_{g-1, r+2}\right)$, we get a presentation for $\operatorname{Stab} s\left(v_{0}\right)$ with generators

$$
\left\{B, B_{2}, \ldots, B_{g-1}, A_{1}, \ldots, A_{2 g+r-2},\left(C_{i, j}\right)_{1 \leqslant i, j \leqslant 2 g+r-2, i \neq j}\right\} \cup \psi
$$

and relations $R_{\gamma_{1,2}} \cup R_{g} \cup\left\{\psi^{2} f_{\psi^{2}}\right\} \cup R_{m}$, where

$R_{\gamma_{1,2}}$ are the Gervais relations for $\mathscr{M}\left(F_{\gamma_{1,2}}\right)$;

$R_{g} \quad$ is the relation $C_{1,2}=C_{2,3}$;

$\psi^{2} f_{\psi^{2}}$ is the relation $\psi^{2} C_{1,3}^{-1}$;

$R_{m} \quad$ are the relations "mixing" the generators of $\operatorname{Stab}\left(\gamma_{1,2}\right)$ and $\psi$.

Therefore the isotropy subgroup of the non-separating vertex carries all the Gervais generators and all the Gervais relations for $\mathscr{M}_{g, r}$, with the exception of the generator $B_{1}$ and the relations involving $B_{1}$, that are all braid relations. Moreover, it carries an extra generator, $\psi$, and the relations $\psi^{2} C_{1,3}^{-1}$ and $R_{m}$.

It is not difficult to see that the generator $B_{1}$ is carried by the isotropy subgroup of a representative (chosen disjoint from $s\left(v_{0}\right)$ ) of any separating vertex, and clearly we may include the braid relations involving $B_{1}$, because they are certainly valid in $\mathscr{M}_{g, r}$.

If $(g, r) \neq(2 k, 0)$, then it is easy to check that all the separating vertices do not carry extra generators and relations (see (4.8)), thus we get

$$
\mathscr{M}\left(F_{g, r}\right)=\left\langle\left\{\begin{array}{c}
\text { Gervais generators } \\
\text { for } F_{g, r}
\end{array}\right\} \cup \psi \mid\left\{\begin{array}{c}
\text { Gervais relations } \\
\text { for } F_{g, r}
\end{array}\right\} \cup\left\{\psi^{2} C_{1,3}^{-1}\right\} \cup R_{m}\right\rangle .
$$

For $\psi$ we may always take the expression $\psi=\left(C_{1,2}^{2} B_{1}\right)^{3}$. Hence, the relation $\psi^{2} C_{1,3}^{-1}$ becomes $\left(C_{1,2}^{2} B_{1}\right)^{6} C_{1,3}^{-1}$, that is a star, and follows from the Gervais relations. The relations $R_{m}$ are of two types:

$$
\psi C=C \psi
$$

for $C=C_{1,2}$ and for each $C$ among the Gervais generators of $\mathscr{M}\left(F_{\gamma_{1,2}}\right)$ such that the corresponding curve $c$ does not intersect $\gamma_{1,3}$;

$$
\psi C \psi^{-1}=T_{\psi(c)},
$$




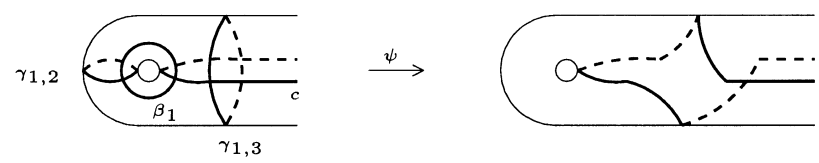

Figure 7.2. The action of $\psi$ on a curve $c$ intersecting $\gamma_{1,3}$.

for each $C$ among the Gervais generators of $\mathscr{M}\left(F_{\gamma_{1,2}}\right)$ such that the corresponding curve $c$ intersects $\gamma_{1,3}$, where $T_{\psi(c)}$ denotes the expression, in terms of the generators of $\mathscr{M}\left(F_{\gamma_{1,2}}\right)$, for the Dehn twist along the curve $\psi(c)$. As far as (7.1) is concerned, it is easy to verify that they always disappear, once we substitute the expression for $\psi$.

Regarding (7.2), notice that the curve $\psi(c)$ is the one depicted in Figure 7.2, and the expression for the Dehn twist along it may always be found by means of a suitable lantern relation: the result has always the form $C^{-1} \cdot\left(\right.$ something) $\cdot C_{1,3}^{-1}$. Thus, using the relation $\psi^{2}=C_{1,3}$, relation (7.2) becomes

$$
C \psi C \psi=\text { (something), }
$$

where the expression we indicated with "something" is in fact a product

$$
C_{1,2}^{2} \cdot\left(\text { an extra star, involving } C, C_{1,2} \text { and a } B_{i}\right) \text {. }
$$

It is easy to verify that the relations (7.3) disappear once we substitute the expression for $\psi$, and we may conclude noticing that the extra star relation always follows from one of those considered by Gervais, as they cover all the possible situations, up to $\mathscr{M}_{g, r}$-equivalence.

If $g=2 k$ and $r=0$, i.e. if our surface is closed and of even genus, the previous argument works, except that we need to take care of the symmetric separating vertex $v_{k, 1}$, that is the vertex separating $F_{2 k, 0}$ into two connected components, both homeomorphic to $F_{k, 1}$. Such a vertex carries in fact a priori a "new" generator, the $\rho$ in (4.7), and the corresponding relations $\rho^{2}$ and $R_{m}$. In this case, we may always take

$$
\rho=A_{1} B A_{2} B_{1} C_{2,4} B_{2} \ldots B_{g-1} C_{2 g-2,1} A_{1} B \ldots C_{2 g-4,2 g-2} B_{g-1} A_{1} \ldots A_{1} B A_{1},
$$

and it is easy to show that the extra relations actually follow from the Gervais ones, once we substitute this expression for $\rho$ in terms of Dehn twists.

Remark 24. The proof we gave is for the case $g \geqslant 2$. The proof for the case of genus 1 , that we omit, uses essentially the same kind of arguments, with some minor difference while considering the isotropy subgroup of the non-separating vertex, due to the fact that in this case the surface $F_{S\left(v_{0}\right)}$ has genus 0 , and therefore we need to consider for its mapping class group the standard presentation, instead of the Gervais one.

\section{Appendix}

We recall here the definition of some basic elements of the (punctured) mapping class group, the Dehn twists, the semitwists and the braid twists, which play an important 

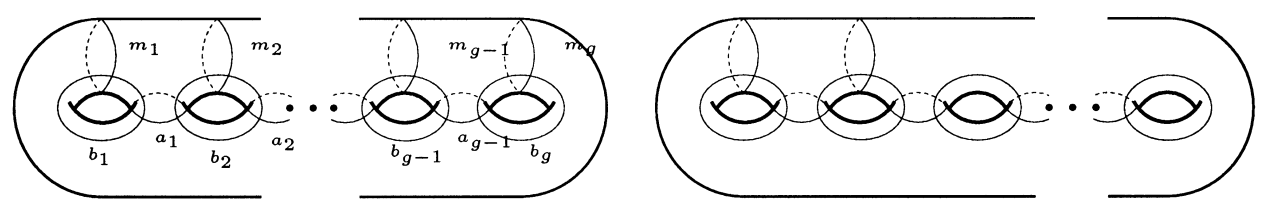

Figure 7.3. Dehn twists generating $\mathscr{M}\left(F_{g, 0}^{0}\right)$.

role in its description: namely, Dehn [5] showed that $\mathscr{M}_{g, 0}^{0}$ is generated by finitely many Dehn twists along certain simple closed curves in $F$, Lickorish [21] proved that it is sufficient to take the $3 g-1$ curves indicated in the left-hand side of Figure 7.3, and Humphries [16] showed that the minimal number of Dehn twist generators for $\mathscr{M}_{g, 0}^{0}$ is $2 g-1$ and proved that one such minimal set is given by the Dehn twists along the curves depicted in the right-hand side of Figure 7.3. Moreover, all the presentations for the mapping class group of non-punctured surfaces we met along the paper are given in terms of Dehn twists.

The braid twists appear when we consider punctured surfaces: for example the generators of the mapping class groups of the punctured sphere and of the punctured disk are certain braid twists. Moreover they are, together with suitable Dehn twists, the generators in the Labruère-Paris presentation of $\mathscr{M}\left(F_{g, r}^{s}\right)$ (see [20]).

About the semitwists, that we met in Section 4 among the generators for the isotropy subgroup of some vertex, we recall that in [30] one such element is proven to be the generator of the center of $\mathscr{M}\left(F_{1,1}^{0}\right)$.

Let $F=F_{g, r}^{s}$ be a surface, and $P$ its set of punctures.

Definition 25. Let $c: \mathbb{S}^{1} \rightarrow F \backslash P$ be a simple closed curve not bounding a disk or a 1-punctured disk (i.e. an essential or generic circle). We choose an embedding $A: \mathbb{S}^{1} \times[0,1] \rightarrow F \backslash P$ of the annulus such that $A(z, 1 / 2)=c(z)$, for all $z \in \mathbb{S}^{1}$, and we consider the homeomorphism $\tau_{c} \in \mathscr{H}(F, P)$ defined by

$$
\begin{cases}\tau_{c} \circ A(z, t)=A\left(e^{2 \pi i t} z, t\right) & t \in[0,1], z \in \mathbb{S}^{1}, \\ \tau_{c}=\text { identity } & \text { outside the image of } A .\end{cases}
$$

We define the Dehn twist along $c$ to be the isotopy class $T_{c}$ in $\mathscr{M}(F, P)$ represented by $\tau_{c}$ (see Figure 7.4).

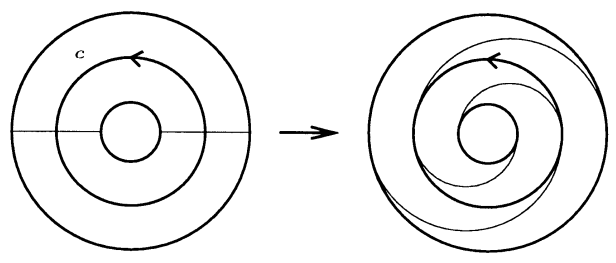

Figure 7.4. Dehn twist along $c$. 
We remark that:

- the element $T_{c}$ does not depend on the choice of $A$, nor on the orientation of $c$;

- if $c$ and $d$ are isotopic, then $T_{c}=T_{d}$;

- if $c$ bounds a disk in $F$ containing exactly one puncture, then $T_{c}=1$. Otherwise, it has infinite order;

- the curve $c$ is fixed by the Dehn twist $T_{c}$;

- if $h$ is in $\mathscr{H}(F, P)$, then $T_{h(c)}=h T_{c} h^{-1}$.

We define an arc to be an embedding $a:[-1 / 2,1 / 2] \rightarrow F$ whose image lies in the interior of $F$, such that $a((-1 / 2,1 / 2)) \cap P=\varnothing$ and $a(-1 / 2), a(1 / 2) \in P$. Two $\operatorname{arcs} a$ and $b$ are called isotopic if there exists $h \in \mathscr{H}(F, P)$, isotopic to the identity, such that $h \circ a=b$; we write in this case $a \simeq b$.

Definition 26. Let $a$ be an arc. We choose an embedding $A$ of the unit disk $D^{2}$ of $\mathbb{C}$ in $F$ such that $a(t)=A(t)$ for every $t \in[-1 / 2,1 / 2]$ (seeing this interval as a subset of $D^{2}$ ), and $A\left(D^{2}\right) \cap P=\{a(-1 / 2), a(1 / 2)\}$. We define a homeomorphism $\tau_{a} \in \mathscr{H}(F, P)$ by

$$
\begin{cases}\tau_{a} \circ A(z)=A\left(e^{2 \pi i|z|} z\right) & z \in D^{2} \\ \tau_{a}=\text { identity } & \text { outside the image of } A .\end{cases}
$$

The braid twist along $a$ is defined to be the isotopy class $T_{a}$ in $\mathscr{M}(F, P)$ represented by $\tau_{a}$ (see Figure 7.5).

We notice that:

- the element $T_{a}$ does not depend on the choice of $A$;

- if $a$ and $b$ are isotopic, then $T_{a}=T_{b}$;

- if $h$ is in $\mathscr{H}(F, P)$, then $T_{h(a)}=h T_{a} h^{-1}$;

- if $c: \mathbb{S}^{1}: \rightarrow F \backslash P$ is the essential circle defined by $c=A_{\mid \mathbb{S}^{1}}$, then $\left(T_{a}\right)^{2}=T_{c}$.

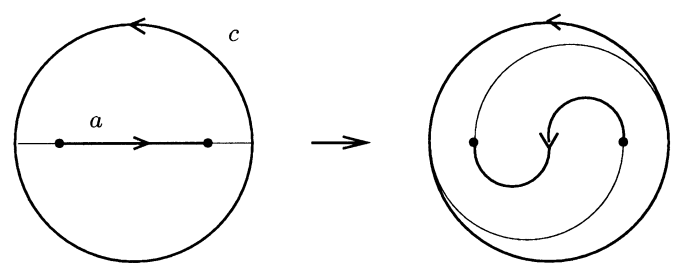

Figure 7.5. Braid twist along $a$. 
Finally, let us consider

$$
\begin{aligned}
D & =\{z \in \mathbb{C}|| z \mid \leqslant 4\}, \\
D_{1} & =\{z \in \mathbb{C}|| z-2 \mid<1\}, \\
D_{2} & =\{z \in \mathbb{C}|| z+2 \mid<1\} .
\end{aligned}
$$

Then $D \backslash\left(D_{1} \cup D_{2}\right)$ is a pair of pants of type III, whose boundary curves $c, a_{1}, a_{2}$ may be parametrized as follows:

$$
\begin{aligned}
c\left(e^{i \theta}\right) & =4 e^{i \theta}, \\
a_{1}\left(e^{i \theta}\right) & =2+e^{i \theta}, \\
a_{2}\left(e^{i \theta}\right) & =-2-e^{i \theta}
\end{aligned}
$$

for $0 \leqslant \theta \leqslant 2 \phi$. We may then consider

$$
F=\left(D \backslash\left(D_{1} \cup D_{2}\right)\right) /\left\{a_{1}\left(e^{i \theta}\right)=a_{2}\left(e^{i \theta}\right)\right\}
$$

We denote by $p$ the natural projection $p: D \backslash\left(D_{1} \cup D_{2}\right) \rightarrow F$, and we define the meridian curve $a: \mathbb{S}^{1} \rightarrow F$ by

$$
a=p \circ a_{1}=p \circ a_{2}
$$

(see Figure 7.6).

Definition 27. We define a homeomorphism $S: D \rightarrow D$ by

$$
S\left(r e^{i \theta}\right)= \begin{cases}r e^{i(\theta-\pi)} & \text { if } 0 \leqslant r \leqslant 3 \\ r e^{i(\theta-(r-2) \pi)} & \text { if } 3 \leqslant r \leqslant 4 .\end{cases}
$$

We have then that $S\left(D_{1}\right)=D_{2}, S\left(D_{2}\right)=D_{1}, S \circ a_{1}=a_{2}^{-1}$ and $S \circ a_{2}=a_{1}^{-1}$. Hence, $S$ induces a homeomorphism $\tilde{S}: F \rightarrow F$ such that $\tilde{S} \circ a=a^{-1}$. We call semitwist of $F$ along c relative to $a$ the isotopy class $\sigma$ of $\tilde{S}$ in $\mathscr{M}(F)$.

We remark that, if $T_{c}$ and $T_{a}$ are the Dehn twists along the corresponding curves of $F$, then $\sigma^{2}=T_{c}$ and $\sigma T_{a} \sigma^{-1}=T_{a}$.

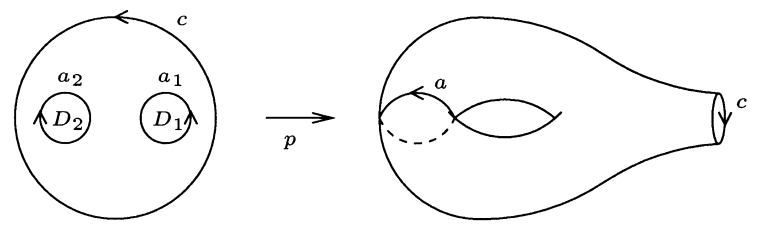

Figure 7.6. The projection map. 
Acknowledgments. This work was partially written during my stay at the Laboratoire de Topologie, Université de Bourgogne, Dijon, in winter 1999/2000. I would like to thank the Laboratoire, in particular Prof. Bernard Perron, for the kind hospitality, and the TMR network "Singularidades de Ecuaciones Differenciales y Foliaciones" for financial support. Also, it is a pleasant duty to thank Prof. Luis Paris, who introduced me to the subject. Finally, I wish to thank Prof. Riccardo Benedetti for many useful discussions and precious advice during the preparation of this work.

\section{References}

[1] J. Birman, Braids, Links, and Mapping Class Groups. Ann. of Math. Studies 82, Princeton University Press, 1975. Zbl 305.57013

[2] T. Brady, Automorphism groups of punctured surfaces. Topology Appl. 55 (1993), 47-66. Zbl 817.20042

[3] M. R. Bridson and A. Haefliger, Metric Spaces of Non-Positive Curvature. Grundl. Math. Wiss. 319, Springer-Verlag, Berlin 1999. Zbl 991.00075

[4] K. S. Brown, Presentations for groups acting on simply-connected complexes. J. Pure Appl. Algebra 32 (1984), 1-10. Zbl 545.20022

[5] M. Dehn, Die Gruppe der Abbildungsklassen. Acta Math. 69 (1938), 135-206. Zbl 019.25301

[6] A. Fathi, F. Laudenbach and V. Poenaru, Travaux de Thurston sur les surfaces. Seminaire Orsay, Asterisque 66-67, 1979.

[7] S. Gervais, A finite presentation of the mapping class group of an oriented surface. To appear in Topology.

[8] S. Gervais, Étude de certaines extensions centrales du "Mapping Class Group" des surfaces orientables. École Doctorale de Mathématiques de l'Ouest, Université de Nantes, 1994.

[9] S. Gervais, Presentation and central extension of mapping class groups. Trans. Amer. Math. Soc. 348 (1996), 3097-3132. Zbl 861.57023

[10] A. Haefliger, Extension of complexes of groups. Ann. Inst. Fourier (Grenoble) 42 (1992), 275-311. Zbl 762.20018

[11] J. Harer, The Second Homology Group of the Mapping Class Group of an Orientable Surface. Invent. Math. 72 (1983), 221-239. Zbl 533.57003

[12] J. Harer, Stability of the homology of the mapping class group of an orientable surface. Ann. of Math. 121 (1985), 215-249. Zbl 579.57005

[13] W. J. Harvey, Boundary structure of the modular group. Ann. Math. Stud. 97, pp. 245-251, Princeton 1981. Zbl 461.30036

[14] A. Hatcher and W. Thurston, A presentation for the mapping class group of a closed orientable surface. Topology 19 (1980), 221-237. Zbl 447.57005

[15] S. Hirose, Action of the mapping class group on a complex of curves and a presentation for the mapping class group of a surface. Preprint, 2000.

[16] S. P. Humphries, Generators for the mapping class group. In: Topology of LowDimensional Manifolds, Proc. Second Sussex Conf., Chelwood Gate, 1977 (R. Fenn, ed.), Lectures Notes in Math. 722, pp. 44-47, Springer-Verlag, Berlin 1979. Zbl 732.57004

[17] N. V. Ivanov, On the virtual cohomology dimension of the Teichmüller modular group. In: Topology, Proceedings, Leningrad 1982 (L. D. Faddeev and A. A. Mal'cev, eds.), Lectures Notes in Math. 1060, pp. 306-318, Springer-Verlag, Berlin 1984. Zbl 583.57006

[18] N. V. Ivanov, Algebraic properties of mapping class groups of surfaces. In: Geometric and algebraic topology, pp. 15-35, PWN, Warsaw 1986. Zbl 635.57004 
[19] N. V. Ivanov, Complexes of curves and Teichmüller modular groups, Uspekhi Mat. Nauk 42 (1987), 49-91, 255. Zbl 637.32021; English translation in Russ. Math. Surv. 42, No. 3, 55-107 (1987).

[20] C. Labruère and L. Paris, Presentation for the punctured mapping class groups in terms of Artin groups. Laboratoire de Topologie, Université de la Bourgogne, Dijon, preprint n. 197, 1999.

[21] W. B. R. Lickorish, A finite set of generators for the homeotopy group of a 2-manifold. Proc. Cambridge Philos. Soc. 60 (1964), 769-778. Zbl 131.20801

[22] W. B. R. Lickorish, On the homeotopy group of a 2-manifold. Proc. Cambridge Philos. Soc. 62 (1966), 679-681. Zbl 145.44102

[23] H. A. Masur and Y. N. Minsky, Geometry of the complex of curves I: Hyperbolicity. Invent. Math. 138 (1999), 103-149. Zbl 941.32012

[24] H. A. Masur and Y. N. Minsky, Geometry of the complex of curves II: Hierarchical structure. Geom. Funct. Anal. 10 (2000), 902-974. Zbl 992.12074

[25] M. Matsumoto, A presentation of mapping class groups in terms of Artin groups and geometric monodromy of singularities. Math. Ann. 316 (2000), 401-418. Zbl 991.60825

[26] J. McCool, Some finitely presented subgroups of the automorphism group of a free group. J. Algebra 35 (1975), 205-213. Zbl 325.20025

[27] Y. N. Minsky, A geometric approach to the complex of curves. In: Proceedings of the 37th Taniguchi Symposium on Topology and Teichmüller Spaces (S. Kojima et al., eds.), pp. 149-158, World Scientific, Singapore 1996. Zbl 937.30027

[28] Y. N. Minsky, Kleinian groups and the complex of curves. Geom. Topol. 4 (2000), 117-148. Zbl 953.30027

[29] L. Paris, Actions and irreducible representations of the mapping class group. Laboratoire de Topologie, Université de la Bourgogne, Dijon, preprint n. 183, 1999.

[30] L. Paris and D. Rolfsen, Geometric subgroups of mapping class groups. J. Reine Angew. Math. 521 (2000), 47-83. Zbl 991.54255

[31] B. Wajnryb, A simple presentation for the mapping class group of an orientable surface. Israel J. Math. 45 (1983), 157-174. Zbl 533.57002

[32] B. Wajnryb, An elementary approach to the mapping class group of a surface. Geom. Topol. 3 (1999), 405-466. Zbl 947.57015

Received 23 November, 2000

S. Benvenuti, Dipartimento di Matematica, Università di Pisa, Via Buonarroti 2, 56100 Pisa, Italy

E-mail: benvenut@dm.unipi.it 